\title{
ON CERTAIN RECIPROCITY-LAWS FOR THETA FUNCTIONS AND MODULAR FORMS
}

\author{
BY \\ GORO SHIMURA( ${ }^{(1)}$ \\ Princeton University, Princeton, U.S.A.
}

The present paper has several objectives. The first apparent theme is an investigation of holomorphic functions $f(u, z)$ on $\mathrm{C}_{s}^{n} \times \mathfrak{Y}_{n}$ which generalize the classical theta function

$$
\theta(u, z)=\sum_{x \in \mathbf{Z}^{n}} \exp \left(\pi i\left({ }^{t} x z x+2 \cdot{ }^{t} x u\right)\right) \quad\left(u \in \mathbf{C}_{1}^{n}, z \in \mathfrak{S}_{n}\right),
$$

where $\mathfrak{S}_{n}$ is the Siegel upper space of degree $n$, and $\mathbf{C}_{s}^{n}$ the vector space of all $n \times s$ complex matrices. As is well known, $\theta$ satisfies a transformation formula under $S p(n, \mathbf{Z})$, and also another formula under a translation $u \mapsto u+z a+b$ with $a, b$ in $\mathbf{Z}^{n}$. Generalizations of these two formulas are the conditions we impose on $f$. Multiplying $f$ by a certain exponential factor, we associate with $f$ a non-holomorphic function $f_{*}(u, z)$ whose value at a point $u=z p+q$ is a holomorphic modular form on $\mathfrak{H}_{n}$ if $p$ and $q$ belong to $\mathbf{Q}_{s}^{n}$, the set of $\mathbf{Q}$-rational elements of $\mathbf{C}_{s}^{n}$. In the special case $f=\theta$, we have

$$
\theta_{*}(u, z)=\exp \left(\pi i \cdot{ }^{t}(u-\bar{u})(z-\bar{z})^{-1} u\right) \theta(u, z) .
$$

Now we consider the group $G$ of similitudes of an alternating form and its restricted adelization $G_{\mathrm{A}+}$, the restriction to the identity component being made at the archimedean place. In order to deal with the modular forms of half integral weight, we introduce a certain covering (BS of $G_{\mathbf{A}+}$, which is modelled on the metaplectic group of Weil [10]. Then we define the action of every element of $\left(B S\right.$ both on modular forms on $\mathscr{S}_{n}$ and on the functions $f(u, z)$ with cyclotomic Fourier coefficients so that a reciprocity-law

$$
f_{*}\left(\Omega_{z} v, z\right)^{y}=\left(f^{y}\right)_{*}\left(\Omega_{z} \cdot{ }^{t} x v, z\right)
$$

holds for all $y \in(S)$ and all $v \in \mathbf{Q}_{s}^{2 n}$, where $\Omega_{z}=\left(z \mathbf{1}_{n}\right)$ and $x$ is the projection of $y$ to $G_{\mathbf{A}+} ; x$ can replace $y$ if the forms are of integral weight (Theorem 3.10). The action of $G_{\mathrm{A}+}$ or (B)

(1) Supported by NSF Grant MCS 76-11376. 
on modular forms is consistent with the concept of canonical models in the sense of [4]. Formula (1) may be considered a "generic form" of the main theorem of complex multiplication of abelian varieties or of Siegel modular functions, which was originally proved without theta functions, and then formulated in terms of theta functions in our recent paper [7]. In fact, (1) enables us to give a simplified proof of the main theorem of [7].

The first two sections of the present paper are devoted to the action of $G_{A+}$ and (S) on modular forms on $\mathfrak{H}_{n}$, which may be of independent interest, though this part is preliminary to the rest of the paper. Formula (1) and its "specializations" will be proved in $\S 3$. The next two sections are of technical nature; the proofs of some statements of the previous sections will be completed there. In $\S 6$, we first observe that if $F$ is a modular form on $\mathfrak{S}_{n+s}$ and if a point $Z$ of $\mathfrak{S}_{n+s}$ is expressed in the form

$$
Z=\left(\begin{array}{ll}
z & u \\
t_{u} & w
\end{array}\right)
$$

with $u \in \mathbb{C}_{s}^{n}, z \in \mathfrak{H}_{n}$, and $w \in \mathfrak{H}_{s}$, then $F$ has a Fourier expansion

$$
F(Z)=\sum_{\xi} f_{\xi}(u, z) \exp (\pi i \cdot \operatorname{tr}(\xi z))
$$

whose Fourier coefficients $f_{\xi}$ belong to the functions of the above type, where $\xi$ runs over non-negative rational symmetric matrices of degree $n$. We shall then prove another reciprocity-law concerning the action of $\left(\mathcal{G}\right.$ and its counterpart $\mathfrak{S}^{\prime}$ of degree $n+s$ on $f_{\xi}$ and $F$ (Theorem 6.2).

We shall present all these for holomorphic modular forms with a rather general automorphic factor $\operatorname{det}(c z+d)^{k / 2} \varrho(c z+d)$, where $k \in \mathbf{Z}$ and $\varrho$ is an arbitrary rational representation of $G L_{n}$. The "theta functions" $f$ will also be defined relative to a representation $\tau$ of $G L_{n+s}$. The consideration of an arbitrary $\varrho$ or $\tau$ is made not merely for the sake of generality, but because there are good reasons for believing that such is natural and even necessary for the future development of the arithmetic theory of modular forms and zeta functions. It should be pointed out, however, that the nature of the reciprocity-laws is essentially revealed in the case of trivial $\varrho$ and trivial $\tau$, and therefore if the reader wishes to have a quick grasp of the ideas, he may be advised to assume throughout $\varrho$ and $\tau$ to be trivial. In fact we have stated (1) in that special case.

While our theory may be accepted on its own merits, it has one significant and hidden aspect. Expansion (2) is actually an example of Fourier-Jacobi series in the sense of Pyatetskii-Shapiro [3]. Such a series occurs naturally as a Fourier expansion of an automorphic form on a Siegel domain of the third kind. When the discontinuous group is arithmetically defined, one can ask whether there is a natural class of "arithmetic auto- 
morphic forms" which is characterized by some properties of Fourier coefficients and which plays the role similar to that of the elliptic modular forms with algebraic or cyclotomic Fourier coefficients. Now our results, especially the above two laws of reciprocity, seem to suggest an affirmative answer to this question. A detailed explanation of the ideas about this is another of our main purposes of this paper, and will be given in the last section, to which the preceding sections may serve as a long introduction.

Notation. For a ring $X$ with an identity element, the set of all $r \times s$ matrices with coefficients in $X$ is denoted by $X_{s}^{r}$, and simply by $X^{r}$ if $s=1$; the identity element of $X_{s}^{s}$ is denoted by $1_{s}$. Further $X^{\times}$denotes the group of all invertible elements of $X$. The diagonal matrix with diagonal elements $d_{1}, \ldots, d_{n}$ is denoted by

$$
\operatorname{diag}\left[d_{1}, \ldots, d_{n}\right]
$$

For $T \in \mathbf{C}_{s}^{s}$, we put

$$
e_{s}(T)=\exp (2 \pi i \cdot \operatorname{tr}(T))
$$

and especially $e(u)=e_{1}(u)=e^{2 \pi t u}$ for $u \in \mathbf{C}$. If $T$ is a hermitian matrix, we write $T \geqslant 0$ or $T>0$ according as $T$ is non-negative or positive definite. The Siegel upper space of degree $n$ is denoted by $\mathfrak{S}_{n}$, thus

$$
\mathfrak{S}_{n}=\left\{z \in \mathbf{C}_{n}^{n} \mid t_{z=z,} \operatorname{Im}(z)>0\right\}
$$

We put $\Omega_{z}=\left(\begin{array}{ll}z & 1_{n}\end{array}\right)$ for $z \in \mathfrak{S}_{n}$.

If $K$ is an algebraic number field, $K_{\mathrm{A}}$ denotes the ring of adeles of $K, K_{\mathrm{A}}^{\times}$the group of ideles of $K$, and $K_{\mathrm{ab}}$ the maximal abelian extension of $K$. By class field theory, every element $y$ of $K_{\mathbf{A}}^{\times}$acts on $K_{\mathrm{ab}}$ as an automorphism. We denote by $a^{y}$ the image of $a \in K_{\mathrm{ab}}$ under $y$. In particular, if $K=\mathbf{Q}$, we put, as usual, $\mathbf{Q}_{\mathbf{A}}=\mathbf{A}$ and $\mathbf{Q}_{\mathbf{A}}^{\times}=\mathbf{A}^{\times}$; further, we denote by $\mathbf{A}_{\mathbf{f}}$ the non-archimedean part of $\mathbf{A}$, and by $\mathbf{A}_{+}^{\times}$the subgroup of $\mathbf{A}^{\times}$consisting of the elements whose archimedean components are positive. For $0<N \in \mathbf{Z}$ and $x, y \in \mathbf{A}_{s}^{s}$, we write $x \equiv y(\bmod N)$ if $x_{p}-y_{p} \in N\left(\mathbf{Z}_{p}\right)_{s}^{s}$ for all primes $p$, where $x_{p}$ and $y_{p}$ are the $p$-components of $x$ and $y$, respectively. For $b \in \mathbf{A}^{\times}$, we denote by $|b|$ the positive rational number such that $|b| \mathbf{Z}_{p}=b_{p} \mathbf{Z}_{p}$ for all $p$. We define a compact subgroup $\mathbf{Z}_{\mathbf{f}}^{\times}$of $\mathbf{A}^{\times}$by $\mathbf{Z}_{\mathbf{f}}^{\times}=\prod_{p} \mathbf{Z}_{p}^{\times}$, where the product is taken over all primes $p$.

\section{The action of $G_{\mathrm{A}^{+}}$on modular forms of integral weight}

Throughout the first six sections, we let $G$ denote the algebraic subgroup of $G L_{2 n}$ defined over $\mathbf{Q}$ such that

$$
G_{\mathbf{Q}}=\left\{\left.\alpha \in G L_{2 n}(\mathbf{Q})\right|^{t} \alpha J \alpha=\nu(\alpha) J \quad \text { with } \nu(\alpha) \in \mathbf{Q}\right\}
$$


where

$$
J=\left(\begin{array}{cc}
0 & -\mathbf{l}_{n} \\
\mathbf{l}_{n} & 0
\end{array}\right)
$$

and $G_{\mathbf{A}}$ its adelization. The $\operatorname{map} v: G_{\mathbf{Q}} \rightarrow \mathbf{Q}^{\times}$can be naturally extended to a continuous map of $G_{\mathrm{A}}$ into $\mathbf{A}^{\times}$, which is still denoted by $\nu$. We denote by $G_{\mathrm{f}}, G_{\infty}$, and $G_{\infty+}$ the nonarchimedean part of $G_{\mathbf{A}}$, the archimedean part of $G_{\mathbf{A}}$, and the identity component of $G_{\infty}$, respectively. We then put $G_{\mathbf{A +}}=G_{\mathbf{P}} G_{\infty+}$ and

$$
G_{\mathbf{Q}+}=G_{\mathbf{A}+} \cap G_{\mathbf{Q}}=\left\{\alpha \in G_{\mathbf{Q}} \mid \nu(\alpha)>0\right\}
$$

Every element $\alpha=\left(\begin{array}{ll}a & b \\ c & d\end{array}\right)$ of $G_{\mathbf{Q}_{+}}$acts on $\mathfrak{S}_{n}$ by the rule $\alpha(z)=(a z+b)(c z+d)^{-1}$ for $z \in \mathfrak{S}_{n}$. Now we take an arbitrary rational representation

$$
\varrho: G L_{n}(\mathbf{Q}) \rightarrow G L_{m}(\mathbf{Q})
$$

and extend it to a holomorphic representation $G L_{n}(\mathbf{C}) \rightarrow G L_{m}(\mathbf{C})$, which is again denoted by $\varrho$. For $\alpha=\left(\begin{array}{ll}a & b \\ c & d\end{array}\right) \in G_{\mathbf{Q}_{+}}$and a $\mathbf{C}^{m}$-valued function $f$ on $\mathfrak{S}_{n}$, we define a function $\left.f\right|_{\varrho} \alpha$ on $\mathfrak{S}_{n}$ by

$$
\left(\left.f\right|_{\varrho} \alpha\right)(z)=\varrho(c z+d)^{-1} f(\alpha(z)) \quad\left(z \in \mathfrak{S}_{n}\right) .
$$

Given a congruence subgroup $\Gamma$ of $G_{\mathbf{Q}_{+}}$, we denote by $m_{\varrho}(\Gamma)$ the vector space of all $\mathbf{C}^{m}$ valued holomorphic functions $f$ on $\mathfrak{S}_{n}$ which satisfy $\left.f\right|_{\varrho} \gamma=f$ for all $\gamma \in \Gamma$ and which are finite at cusps. Such $f$ may be called modular forms of weight $\varrho$. If $f \in m_{\varrho}(\Gamma), f$ has a Fourier expansion

$$
f(z)=\sum_{\xi} c(\xi) e_{n}(\xi z)
$$

with $c(\xi) \in \mathbf{C}^{m}$, where $\xi$ runs over positive semi-definite symmetric elements of $\mathbf{Q}_{n}^{n}$. If $n>1$, every holomorphic $f$ satisfying $\left.f\right|_{\varrho} \gamma=f$ for $\gamma \in \Gamma$ has such an expansion, and hence belongs to $m_{\varrho}(\Gamma)$ (see for example [1]). For a subfield $\mathfrak{R}$ of $\mathbf{C}$, we denote by $m_{\varrho}(\Gamma, \Re)$ the set of all $f$ in $m_{\varrho}(\Gamma)$ with $c(\xi)$ in $\Re^{m}$, and by $m_{\varrho}(\mathfrak{R})$ the union of $m_{\varrho}(\Gamma, \mathfrak{R})$ for all congruence subgroups $\Gamma$. Further we denote by $\mathcal{A}_{\varrho}(\Re)$ the set of all quotients $g^{-1} h$ with $h \in m_{\tau}(\Re)$ and $0 \neq g \in m_{\omega}(\Re)$, where $\omega(X)=\operatorname{det}(X)^{k}, \tau=\omega \varrho$ with some integer $k$. Then we put

$$
\mathcal{A}_{\varrho}(\Gamma, \Re)=\left\{f \in \mathcal{A}_{\varrho}(\Re)|\quad f|_{\varrho} \gamma=f \quad \text { for all } \gamma \in \Gamma\right\} \text {. }
$$

It can easily be shown that $\mathcal{M}_{e}(\Gamma, \Re)$ consists of all the holomorphic elements of $\mathcal{A}_{Q}(\Gamma, \Re)$ finite at cusps. If $\varrho(X)=\operatorname{det}(X)^{k}$ with $k \in \mathbf{Z}$, we write $\left.f\right|_{k} \alpha, m_{k}, \mathcal{A}_{k}$ for $f_{\varrho} \alpha, m_{\varrho}, \mathcal{A}_{\varrho}$. 
Let $\sigma$ be a field-automorphism of $\mathbf{C}$. Define the action of $\sigma$ on $\mathbf{C}^{m}$ component-wise. For $f \in m_{\varrho}(\mathbf{C})$ with expansion (1.3), we define $f^{\sigma}$ by

$$
f^{\sigma}(z)=\sum_{\xi} c(\xi)^{\sigma} e_{n}(\xi z)
$$

If $\varrho(X)=\operatorname{det}(X)^{k}, f^{\sigma}$ is actually an element of $m_{k}(\mathbf{C})$; moreover $\sigma$ can be naturally extended to an automorphism of $\mathcal{A}_{k}(\mathbf{C})$ (see $[6, \S 4]$ ). We shall prove in $\S 5$ that these hold for an arbitrary $\varrho$. The notation $f^{\sigma}$ is meaningful if $\sigma$ is an isomorphism of a subfield $\mathfrak{R}$ of $\mathbf{C}$ onto another subfield and $f \in \mathcal{A}_{\varrho}(\Re)$; in particular $f^{t}$ is meaningful for $t \in \mathbf{A}^{\times}$if $f \in \mathcal{A}_{\varrho}\left(\mathbf{Q}_{\mathrm{ab}}\right)$.

Now we define an injection $\imath: \mathbf{A}_{+}^{\times} \rightarrow G_{\mathbf{A}_{+}}$by

$$
\iota(t)=\left(\begin{array}{cc}
1_{n} & 0 \\
0 & t^{-1} 1_{n}
\end{array}\right) \quad\left(t \in \mathbf{A}_{+}^{\times}\right)
$$

In our treatment, we shall often need the strong approximation theorem in $G_{\mathrm{A}}$, which can be given as

LEM M 1.1. Let $T$ be an open subgroup of $\left\{x \in G_{\mathbf{A}} \mid v(x)=1\right\} G_{\infty+}$. Then $G_{\mathbf{A}+}$ is the product of $G_{\mathbf{Q}_{+}, \iota}\left(\mathbf{Z}_{\mathbf{i}}^{\times}\right)$, and $T$ in an arbitrary order, i.e., $G_{\mathbf{A}_{+}}=G_{\mathbf{Q}_{+}} \iota\left(\mathbf{Z}_{\mathbf{i}}^{\times}\right) T=T_{\iota}\left(\mathbf{Z}_{\mathfrak{\ell}}^{\times}\right) G_{\mathbf{Q}_{+}}=\cdots$.

Cf. [4, I, 3.4; II, (3.10.3)].

We are going to define the action of $G_{\mathbf{A}+}$ on $\mathcal{A}_{Q}\left(\mathbf{Q}_{\mathrm{ab}}\right)$. First we recall that $G_{\mathbf{A}+}$ acts on the field $\mathcal{A}_{0}\left(\mathbf{Q}_{\mathrm{ab}}\right)$ as a group of automorphisms, and for every $t \in \mathbf{A}_{+}^{\times}$, the action of $\iota(t)$ on $\mathbf{Q}_{\mathrm{ab}}$ is the same as that of $t$ (see [4], [5], [6]).

THEOREM 1.2. There is an action of $G_{\mathrm{A}+}$ on $\mathcal{A}_{\ell}\left(\mathbf{Q}_{\mathrm{ab}}\right)$, written as $(x, f) \mapsto f^{x}$ for $x \in G_{\mathrm{A}+}$ and $f \in \mathcal{A}_{Q}\left(\mathbf{Q}_{\mathrm{ab}}\right)$, with the following properties:

(i) $m_{\varrho}\left(\mathbf{Q}_{\mathrm{ab}}\right)$ is stable under the action;

(ii) the action of $G_{\mathbf{A}+}$ on $A_{0}\left(\mathbf{Q}_{\mathrm{ab}}\right)$ is the same as that mentioned above;

(iii) the action is associative, i.e., $f^{x y}=\left(f^{x}\right)^{y}$;

(iv) $(f \oplus g)^{x}=f^{x} \oplus g^{x},(f \otimes g)^{x}=f^{x} \otimes g^{x}$, where $f \oplus g$ and $f \otimes g$ are naturally defined as elements of $\mathcal{A}_{\varrho \oplus \tau}\left(\mathbf{Q}_{\mathrm{ab}}\right)$ and $\mathcal{A}_{\varrho \otimes \tau}\left(\mathbf{Q}_{\mathrm{ab}}\right)$ for $f \in \mathcal{A}_{\varrho}\left(\mathbf{Q}_{\mathrm{ab}}\right)$ and $g \in \mathcal{A}_{\tau}\left(\mathbf{Q}_{\mathrm{ab}}\right)$;

(v) $f^{\alpha}=\left.f\right|_{e} \alpha$ if $\alpha \in G_{Q_{+}}$;

(vi) $f^{(t)}=f^{t}$ for $t \in \mathbf{Z}_{\mathbf{f}}^{\times}$;

(vii) $f^{x}=f$ if $x \in G_{\infty+}$;

(viii) for each $f \in \mathcal{A}_{\varrho}\left(Q_{\mathrm{ab}}\right)$, the element $x$ of $G_{\mathbf{A}+}$ such that $f^{x}=f$ form an open subset of $G_{\mathbf{A}+}$.

This extends the previous result [6, Theorem 5], which concerns the action of $G_{\mathbf{A}+}$ on $\mathcal{A}_{k}\left(\mathbf{Q}_{\mathrm{ab}}\right)$ with $k \in 2 Z$. In the previous papers, we defined $\left.f\right|_{k} \alpha$ for $\alpha \in G_{\mathbf{A}+}$ with a scalar 
factor $v(\alpha)^{k / 2}$, which is different from our present definition (1.2). This change is necessary to guarantee (iii) in the general case. Before proving our theorem, we first state the uniqueness of the action as

Proposition 1.3. The action of $G_{\mathbf{A}+}$ on $\mathcal{A}_{Q}\left(\mathbf{Q}_{\mathrm{ab}}\right)$ is uniquely determined by (iii), (v), (vi), (viii).

Proof. Let $U=\left\{x \in G_{\mathrm{A}_{+}} \mid f^{x}=f\right\}$ with any given $f$. By Lemma 1.1, if $U$ is open, each element $x$ of $G_{\mathbf{A}+}$ can be written as $x=u \iota(t) \alpha$ with $u \in U, t \in \mathbf{Z}_{\mathbf{f}}^{\times}$and $\alpha \in G_{\mathbf{Q}_{+}}$. Therefore, assuming (iii), (v), (vi), we have $f^{x}=\left.f^{t}\right|_{\varrho} \alpha$, which proves our assertion.

In this section, we prove our theorem only for $\mathcal{A}_{k}\left(\mathbf{Q}_{\mathrm{ab}}\right)$ with $k \in \mathbf{Z}$. The general case will be discussed in $\S 5$. First put

$$
\theta(z)=\sum_{v \in \mathbb{Z}^{n}} e\left({ }^{t} v z v / 2\right) \quad\left(z \in \mathfrak{S}_{n}\right)
$$

and for each positive integer $N$,

$$
\begin{gathered}
\Gamma_{N}=\left\{\gamma \in G_{\mathbf{Q}} \cap S L_{2 n}(\mathbf{Z}) \mid \gamma \equiv 1_{2 n}(\bmod N)\right\}, \\
S_{1}=G_{A+} \cap\left\{G_{\infty+} \prod_{p} G L_{2 n}\left(\mathbf{Z}_{p}\right)\right\} \\
S_{N}^{\prime}=\left\{x \in S_{1} \mid x \equiv\left(\begin{array}{rr}
1_{n} & 0 \\
0 & t 1_{n}
\end{array}\right)(\bmod N) \text { with } t \in \mathbf{Z}_{\mathfrak{\ell}}^{\times}\right\} \\
S_{N}=\left\{x \in S_{1} \mid x \equiv 1_{2 n}(\bmod N)\right\} .
\end{gathered}
$$

Obviously it is sufficient to consider the case $k>0$. Fix a positive integer $k$, and put $h(z)=$ $\theta(z)^{2 k}$. As shown in [7], there is a positive integer $M$ such that $h \in m_{k}\left(\Gamma_{M}\right)$. Take any such $M$ that is a multiple of 4 , and put $T=S_{M}^{\prime}$. By Lemma 1.1, every element $x$ of $G_{\mathrm{A}+}$ can be written as $x=u \alpha$ with $u \in T$ and $\alpha \in G_{\mathbf{Q}_{+}}$. Now we have $\mathcal{A}_{k}\left(\mathbf{Q}_{\mathrm{ab}}\right)=h \cdot \mathcal{A}_{0}\left(\mathbf{Q}_{\mathrm{ab}}\right)$. Define the action of $x$ on $\mathcal{A}_{k}\left(\mathbf{Q}_{\mathrm{ab}}\right)$ by $g^{x}=(g / h)^{x}\left(\left.h\right|_{k} \alpha\right)$ for $g \in \mathcal{A}_{k}\left(\mathbf{Q}_{\mathrm{ab}}\right)$. By [6, Th. 4] or [7, Prop. 1.5], $\left.h\right|_{k} \alpha \in M_{k}\left(\mathbf{Q}_{\mathrm{ab}}\right)$, so that $g^{x} \in \mathcal{A}_{k}\left(\mathbf{Q}_{\mathrm{ab}}\right)$. We see easily that this does not depend on the choice of $u$ and $\alpha$. Also we have obviously $g^{\alpha}=\left.g\right|_{k} \alpha, g^{\alpha \beta}=\left(g^{\alpha}\right)^{\beta}, g^{u v}=\left(g^{u}\right)^{v}, g^{u \alpha}=\left(g^{u}\right)^{\alpha}$ for $\alpha, \beta \in G_{\mathbf{Q}}$ and $u, v \in T$. Assume that

$$
\left(g^{\alpha}\right)^{y}=g^{\alpha y} \quad \text { for } g \in \mathcal{A}_{k}\left(\mathbf{Q}_{\mathrm{ab}}\right), \alpha \in G_{\mathbf{Q}+}, y \in G_{\mathbf{A}+}
$$

holds. Given $x$ and $y$ of $G_{\mathbf{A}+}$, put $x=u \alpha$ as above and $\alpha y=v \beta$ with $v \in T$ and $\beta \in G_{\mathbf{Q}+}$. Then, by (1.8), $\left(g^{x}\right)^{y}=\left(g^{u \alpha}\right)^{y}=\left(g^{u}\right)^{\alpha y}=\left(g^{u}\right)^{v \beta}=\left(g^{u v}\right)^{\beta}=g^{u v \beta}=g^{x y}$. Thus the proof of (iii) can be reduced to (1.8). Observe that if (1.8) is true for some fixed $\alpha, y$, and $g \neq 0$, then it is true for all $g$ in $\mathcal{A}_{k}\left(\mathbf{Q}_{\mathrm{ab}}\right)$ and for the same $\alpha$ and $y$; if (1.8) is true for $\alpha$, then so is for $\alpha^{-1}$. There- 
fore it is sufficient to prove (1.8) for $g=h$ and for $\alpha$ belonging to a set of generators, say $B$, of $G_{\mathrm{Q}_{+}}$. Given $\alpha \in B$ and $y \in G_{\mathrm{A}_{+}}$, put $y=v \beta$ with $v \in T$ and $\beta \in G_{\mathrm{Q}_{+}}$, and $\alpha v=u \gamma$ with $u \in T$ and $\gamma \in G_{\mathbf{Q}_{+}}$. Suppose $\left(h^{\alpha}\right)^{v}=h^{\alpha v}$. Then $\left(h^{\alpha}\right)^{y}=\left(\left(h^{\alpha}\right)^{v}\right)^{\beta}=\left(h^{u \gamma}\right)^{\beta}=\left(\left(h^{u}\right)^{\gamma}\right)^{\beta}=h^{u \gamma \beta}=h^{\alpha y}$. Thus it is sufficient to prove

$$
\left(h^{\alpha}\right)^{v}=h^{\alpha v} \quad \text { for } \alpha \in B \text { and } v \in T \text {. }
$$

Now by [7, Lemma 2], we can take $B$ to be the set consisting of $J$ and the elements of the form $\left(\begin{array}{ll}a & b \\ 0 & d\end{array}\right)$ contained in $Z_{2 n}^{2 n}$. First consider the case $\alpha=J$. If $v \in T$, we have $v \equiv\left(\begin{array}{ll}1 & 0 \\ 0 & t\end{array}\right)(\bmod M)$ with $t \in \mathbf{Z}_{\mathrm{f}}^{\times}$. Take $\left(\begin{array}{ll}p & q \\ r & s\end{array}\right) \in S L_{2}(\mathbf{Z})$ so that $s>0,\left(\begin{array}{ll}p & q \\ r & s\end{array}\right) \equiv\left(\begin{array}{ll}t & 0 \\ 0 & t^{-1}\end{array}\right)(\bmod M)$, and put $\beta=$ $\left(\begin{array}{ll}p 1_{n} & q 1_{n} \\ r 1_{n} & s 1_{n}\end{array}\right), \alpha v=w \beta \alpha$. Then $w \equiv v(\bmod M)$, so that $w \in T$. Hence $h^{\alpha v}=h^{\delta \alpha}$. By Prop. A.2 of the Appendix, we have $h^{\beta}=\left(\frac{-1}{s}\right)^{k n} h$. On the other hand, $h^{\alpha}=(-i)^{k n} h$ by $\left[7,\left(16^{\prime}\right)\right]$ and $i^{v}=\left(\frac{-1}{s}\right) i$, so that $\left(h^{\alpha}\right)^{v}=\left(\frac{-1}{s}\right)^{k n}(-i)^{k n} h=h^{\beta \alpha}=h^{\alpha v}$, which proves (1.9) for $\alpha=J$. Next assume $\alpha=\left(\begin{array}{ll}a & b \\ 0 & d\end{array}\right) \in G_{\mathbf{Q}+} \cap \mathbf{Z}_{2 n}^{2 n}$. Let $N=M \cdot \operatorname{det}(\alpha)$ and put $v=w \beta$ with $w \in S_{N}^{\prime}$ and $\beta \in G_{\mathbf{Q}+}$. Then $w \equiv \iota(t)(\bmod N)$ with $t \in \mathbf{Z}_{\mathrm{f}}^{\times}$. Let $s$ be a positive integer such that $t^{-1} \equiv s(\bmod N)$, and put $\gamma=\left(\begin{array}{ll}a & s b \\ 0 & d\end{array}\right), u=\alpha w \gamma^{-1}$. Then $u \in S_{M}^{\prime}$. Therefore $h^{\alpha v}=h^{u \gamma \beta}=h \gamma \beta$. On the other hand $\left(h^{\alpha}\right)^{v}=\left(h^{\alpha}\right)^{w_{\beta}}=\left(h^{\alpha} / h\right)^{w \beta} h^{\beta}$. Let $h(z)=\sum_{\xi} c(\xi) e_{n}(\xi z)$ be the Fourier expansion of $h$. Then

$$
\begin{aligned}
& h^{\alpha}(z)=\operatorname{det}(d)^{-k} \sum_{\xi} c(\xi) e_{n}\left(\xi b d^{-1}\right) e_{n}\left(d^{-1} \xi a z\right), \\
& h^{\gamma}(z)=\operatorname{det}(d)^{-k} \sum_{\xi} c(\xi) e_{n}\left(s \xi b d^{-1}\right) e_{n}\left(d^{-1} \xi a z\right) .
\end{aligned}
$$

Since $2 \xi \in \mathbf{Z}_{n}^{n}$, we see that $h^{\alpha} \in m_{k}\left(\Gamma_{N}, \mathbf{Q}(e(1 / N))\right)$, and hence $\left(h^{\alpha}\right)^{t}=h^{\gamma}$. By [6, Th. 2, (ii); Th. 3, (i)], we have $\left(h^{\alpha} / h\right)^{w}=\left(h^{\alpha}\right)^{t} / h=h^{\gamma} / h$. Therefore $\left(h^{\alpha}\right)^{v}=h^{\gamma \beta}=h^{\alpha v}$. Thus (1.9) is true for all $\alpha=\left(\begin{array}{ll}a & b \\ 0 & d\end{array}\right)$ and $\alpha=J$. This completes the proof of (iii). Now assertions (ii), (v), (vi), (vii), (viii) are obvious from our definition of the action and [6, Th. 2, (ii)]. If $f \in m_{k}\left(\mathbf{Q}_{\mathrm{ab}}\right)$ and $x \in G_{\mathbf{A}+}, f^{x}=\left(f^{t}\right)^{\alpha}$ with suitable $t$ and $\alpha$ as shown in the proof of Prop. 1.3. This together with [6, Th. 2, Th. 4] proves (i).

The above property (vi) can be generalized as follows:

$$
\left(\sum_{\xi} c(\xi) e_{n}(\xi z)\right)^{\iota(b)}=\varrho\left(|b| \mathbf{l}_{n}\right) \sum_{\xi} c(\xi)^{b} e_{n}(|b| \xi z)
$$


if $b \in \mathbf{A}_{+}^{\times}$and $\sum_{\xi} c(\xi) e_{n}(\xi z) \in M_{\varrho}\left(\mathbf{Q}_{\mathrm{ab}}\right)$. This can be shown by decomposing $b$ as $b=s t u$ with $s=|b|, t \in \mathbf{Z}_{\mathbf{f}}^{\times}$, and $u \in \mathbf{Q}_{\infty+}^{\times}$.

Let $S_{\mathbf{0}}$ be an open compact subgroup of $G_{\mathrm{f}}$, and let $S=S_{\mathbf{0}} G_{\infty+}, \Gamma_{S} \cap G_{\mathbf{Q}}=S$. Further let $k_{S}$ be the subfield of $\mathbf{Q}_{\mathrm{ab}}$ corresponding to the subgroup $\mathbf{Q}^{\times} \boldsymbol{v}(S)$ of $\mathbf{A}^{\times}$. Suppose that the following condition is satisfied:

$$
\iota(t) \in S \quad \text { if } t \in \mathbf{Z}_{\mathrm{f}}^{\times} \text {and } t \text { gives the identity map on } k_{S} .
$$

For example, the above $S_{N}$ and $S_{N}^{\prime}$ satisfy this.

Proposition 1.4. The notation being as above, if (1.11) is satisfied, then $\mathcal{A}_{\varrho}\left(\Gamma_{S}, k_{S}\right)=$ $\left\{f \in \mathcal{A}_{0}\left(\mathbf{Q}_{\mathrm{ab}}\right) \mid f^{x}=f\right.$ for all $\left.x \in S\right\}$.

Proof. Given $f \in \mathcal{A}_{\ell}\left(\Gamma_{S}, k_{S}\right)$ and $x \in S$, put $U=\left\{y \in S \mid f^{y}=f\right\}$ and $x=u \iota(t) \alpha$ with $u \in U$, $t \in \mathbf{Z}_{\mathbf{f}}^{\times}$and $\alpha \in G_{\mathbf{Q}+}$. Then $\iota(t) \in S$ by (1.11), so that $\alpha \in \Gamma_{S}$. Therefore $f$ is invariant under $u$, $\iota(t)$, and $\alpha$, and hence $f^{x}=f$. Conversely, if $f^{x}=f$ for all $x \in S, f$ is invariant under $\Gamma_{S}$ and $\iota\left(\mathbf{Z}_{\mathbf{f}}^{\times}\right) \cap S$, so that $f \in \mathcal{A}_{Q}\left(\Gamma_{S}, k_{S}\right)$, Q.E.D.

\section{The forms of half-integral weight}

The purpose of this section is to define a group (S) acting on the modular forms of halfintegral weight in exactly the same fashion as $G_{\mathbf{A}+}$ on $\mathcal{A}_{\varrho}\left(\mathbf{Q}_{\mathrm{ab}}\right)$. Let $\varrho$ be as in $\S 1$. With $k \in 2^{-1} \mathbf{Z}$ and a subfield $\mathfrak{R}$ of $\mathbf{C}$, we define $\mathcal{A}_{\varrho, k}(\Re)$ to be the set of all functions of the form $\theta^{2 k} f$ with $f \in \mathcal{A}_{\varrho}(\Re)$, where $\theta$ is defined by (1.6). Also we denote by $m_{\varrho, k}(\Re)$ the set of all holomorphic elements of $\mathcal{A}_{\varrho, k}(\Re)$ that are finite at cusps. If $k-l \in \mathbf{Z}$ and $\tau(X)=\operatorname{det}(X)^{k-l} \varrho(X)$, then $\mathcal{A}_{\varrho, k}=\mathcal{A}_{\tau, l}$ and $m_{\varrho, k}=m_{\tau, l}$. If $\varrho$ is trivial, we write $\mathcal{A}_{\varrho, k}$ and $m_{\ell, k}$ simply as $\mathcal{A}_{k}$ and $m_{k}$. The action of an isomorphism $\sigma$ of $\mathfrak{R}$ onto a subfield of $\mathbf{C}$ can be defined on $\mathcal{A}_{\ell, k}(\Re)$ by $f^{\sigma}=\left(f / 0^{2 k}\right)^{\sigma} \theta^{2^{k}}$ for $f \in \mathcal{A}_{\ell, k}(\Re)$. We shall prove in $\S 5$ that $m_{Q, k}(\mathbf{C})=m_{Q, k}(\mathbf{Q}) \otimes \mathbf{Q} \mathbf{C}$ so that $\sigma$ maps $m_{\ell, k}(\mathbf{C})$ into itself; its action is defined again by (1.4).

Let $\mathbb{E}$ denote the group of all $\mathbf{Q}$-linear automorphisms of the module $\mathcal{A}_{1 / 2}\left(\mathbf{Q}_{\mathrm{ab}}\right)$. For any finite subset $F$ of $\mathcal{A}_{1 / 2}\left(\mathbf{Q}_{\mathrm{ab}}\right)$, take

$$
\left\{v \in \mathbb{E} \mid f^{v}=f \text { for all } f \in F\right\}
$$

as a neighborhood of the identity element of $\mathbb{E}$. Then $\mathbb{E}$ becomes a topological group. Let $W$ be the group of all roots of unity. Now we define a subgroup $\mathbb{G S}$ of $G_{\mathbf{A}+} \times \mathbb{E}$ with induced topology by

$$
\begin{gathered}
\left(\mathbb{S}=\left\{(x, v) \in G_{\mathrm{A}+} \times \mathbb{E} \mid\left(f^{v}\right)^{2}=\zeta\left(f^{2}\right)^{x} \quad \text { for all } f \in \mathcal{A}_{1 / 2}\left(\mathbf{Q}_{\mathrm{ab}}\right)\right.\right. \\
\text { with } \zeta \in W \text { independent of } f\} .
\end{gathered}
$$


The root of unity $\zeta$ being determined by $\left(f^{v}\right)^{2}=\zeta\left(f^{2}\right)^{x}$, we shall write $(x, v)$ also as $(x, \zeta, v)$ to emphasize $\zeta$. If $(x, \zeta, v) \in(S)$ and $f, g \in \mathcal{A}_{1 / 2}\left(\mathbf{Q}_{\mathrm{ab}}\right)$, we have $\left((f+g)^{v}\right)^{2}=\zeta\left((f+g)^{2}\right)^{x}$, so that $f^{v} g^{v}=\zeta(f g)^{x}$, and hence

$$
g^{v} / f^{v}=(g / f)^{x} \quad \text { if }(x, v) \in\left(\mathfrak{S} \quad \text { and } f, g \in \mathcal{A}_{1 / 2}\left(\mathbf{Q}_{\mathrm{ab}}\right)\right.
$$

For every $\zeta \in W$, we can define an element of $(\mathcal{E}$ by $f \mapsto \zeta f$, which is denoted again by $\zeta$. Then $\left(1, \zeta^{2}, \zeta\right)$ is an element of (SS. Let $W_{1}$ denote the set of all such elements. Then we see easily that

$$
1 \rightarrow W_{1} \rightarrow \mathbb{G S} \rightarrow G_{\mathrm{A}_{+}} \rightarrow 1
$$

is exact, with a natural projection map of $\left(\mathfrak{G}\right.$ into $G_{\mathbf{A}+}$. In fact, to see the surjectivity, let $U=\left\{y \in G_{\mathbf{A}+} \mid\left(\theta^{2}\right)^{y}=\theta^{2}\right\}$. Given $x \in G_{\mathbf{A}+}$, we have $x=y \iota(t) \alpha$ with $y \in U, t \in \mathbf{Z}_{\mathrm{f}}^{\times}$, and $\alpha=$ $\left(\begin{array}{ll}a & b \\ c & d\end{array}\right) \in G_{\mathbf{Q}+}$ by Lemma 1.1. Define $v \in \mathbb{E}$ by

$$
f^{v}=(f / \theta)^{x} \theta(\alpha(z)) \operatorname{det}(c z+d)^{-1 / 2}
$$

with any choice of $\operatorname{det}(c z+d)^{-1 / 2}$. Then $(x, 1, v) \in(\mathfrak{G}$.

Now we define the action of $\left(\mathfrak{S}\right.$ on $\mathcal{A}_{Q, k}\left(\mathbf{Q}_{\mathrm{ab}}\right)$ as follows. For $(x, v) \in\left(\mathfrak{S}\right.$ and $f \in \mathcal{A}_{Q, k}\left(\mathbf{Q}_{\mathrm{ab}}\right)$, we put

$$
f^{(x, v)}=\left(f / h^{2 k}\right)^{x}\left(h^{v}\right)^{2 k}
$$

with any non-zero $h \in \mathcal{A}_{1 / 2}\left(\mathbf{Q}_{\mathrm{ab}}\right)$. This is independent of the choice of $h$. Moreover $f^{(x, v)}=f^{v}$ if $\varrho$ is trivial and $k=1 / 2$; further, if $(x, \zeta, v) \in\left(\mathscr{S}\right.$ and $k \in \mathbf{Z}$, we have $f^{(x, \zeta, v)}=\zeta^{k} f^{x}$. As mentioned above, $\mathcal{A}_{\varrho, k}\left(\mathbf{Q}_{\mathrm{ab}}\right)=\mathcal{A}_{\tau, l}\left(\mathbf{Q}_{\mathrm{ab}}\right)$ if $\tau(X)=\operatorname{det}(X)^{k-l} \varrho(X)$. But the action of $(x, v)$ on this same set depends on $(\varrho, k)$. To avoid a complicated notation, we hereafter understand that if the set is denoted by $\mathcal{A}_{\varrho, k}\left(\mathbf{Q}_{\mathrm{ab}}\right)$, the action of $(\mathcal{S}$ is defined with the same $\varrho$ and $k$. Notice that the action of $(x, 1, v)$ is independent of $\varrho$ and $k$.

Let $\left(\mathfrak{S}_{\mathbf{Q}}\right.$ denote the set consisting of all pairs $(\alpha, \psi)$ formed by an element $\alpha=\left(\begin{array}{ll}a & b \\ c & d\end{array}\right)$ of $G_{\mathbf{Q}+}$ and a holomorphic function $\psi$ on $\mathscr{H}_{n}$ such that $\psi(z)^{2}=\zeta \cdot \operatorname{det}(c z+d)$ with $\zeta \in W$. A law of composition

$$
(\alpha, \psi(z))\left(\alpha^{\prime}, \psi^{\prime}(z)\right)=\left(\alpha \alpha^{\prime}, \psi\left(\alpha^{\prime}(z)\right) \psi^{\prime}(z)\right)
$$

makes $\mathscr{G}_{\mathbf{Q}}$ a group. For a function $f$ on $\mathfrak{S}_{n}$ and $\beta=(\alpha, \psi) \in \mathfrak{G}_{\mathbf{Q}}$, we define a function $\left.f\right|_{\ell, k} \beta$ by

$$
\left.f\right|_{\varrho, k} \beta=\left(\left.f\right|_{\varrho} \alpha\right) \psi^{-2 k}
$$


By [7, Prop. 1.5], we see easily that $\mathcal{A}_{\ell, k}\left(\mathbf{Q}_{\mathrm{ab}}\right)$ is stable under this action, and hence, in particular, it defines an element of $\mathfrak{E}$. Then

$$
(\alpha, \psi) \mapsto(\alpha,(\alpha, \psi))
$$

gives an embedding of $\mathbb{S}_{\mathbf{Q}}$ into $\mathbb{S S}$. Identify $\mathbb{B}_{\mathbf{Q}}$ with its image in $\mathbb{G}$. Then $\mathbb{G}_{\mathbf{Q}}$ is the inverse image of $G_{\mathbf{Q}_{+}}$, and thus

$$
1 \rightarrow W_{1} \rightarrow \mathscr{G}_{\mathbf{Q}} \rightarrow G_{\mathbf{Q}+} \rightarrow 1
$$

is exact. We see easily that $f^{\beta}=\left.f\right|_{\varrho, k} \beta$ if $\beta \in \mathscr{G}_{\mathbf{Q}}$ and $f \in \mathcal{A}_{\varrho . k}\left(\mathbf{Q}_{\mathrm{ab}}\right)$.

Next, for $t \in \mathbf{Z}_{\mathrm{f}}^{\times}$, denote by $t^{\prime}$ the element of $\mathbb{E}$ given by $f \mapsto f^{t}$ for $f \in \mathcal{A}_{1 / 2}\left(\mathbf{Q}_{\mathrm{ab}}\right)$. Then $\left(\iota(t), t^{\prime}\right) \in(S)$. We see easily that the map $t \mapsto\left(\iota(t), t^{\prime}\right)$ can be extended to an injection $\iota_{1}$ of $\mathbf{A}_{+}^{\times}$into (S) so that

$$
\left(\sum_{\xi} c(\xi) e_{n}(\xi z)\right)^{11^{(b)}}=|b|^{k n} \varrho\left(|b| \mathbf{1}_{n}\right) \sum_{\xi} c(\xi)^{b} e_{n}(|b| \xi z)
$$

if $\sum_{\xi} c(\xi) e_{n}(\xi z) \in m_{\varrho . k}\left(\mathbf{Q}_{\mathrm{ab}}\right)$ and $b \in \mathbf{A}_{+}^{\times}$.

Now we can state the properties of the action of $(S)$ on $\mathcal{A}_{Q, k}\left(\mathbf{Q}_{\mathrm{ab}}\right)$ in exactly the same fashion as in Theorem 1.2 with $\mathbb{S G}_{Q}$ and $\iota_{1}$ in place of $G_{\mathbf{Q}+}$ and $\iota$. In substance, this is already done in the above discussion. (See also Prop. 2.2 below.) We note that if $\Gamma_{\theta}$ is defined by (a.1) of the Appendix, then

$$
\gamma \mapsto(\gamma,(\theta \circ \gamma) / \theta)
$$

defines an injection of $\Gamma_{\theta}$ into $\mathfrak{G}_{\mathbf{Q}}$. By a congruence subgroup of $\mathfrak{G S}_{\mathbf{Q}}$, we understand a subgroup $\Delta$ of $B_{Q}$ such that:

$\left(2.8_{\mathfrak{a}}\right)$ the projection map of $\mathfrak{G}_{\mathbf{Q}}$ to $G_{\mathbf{Q}_{+}}$gives a one-to-one map of $\Delta$ onto a subgroup $\Gamma$ of $\left\{\alpha \in G_{\mathbf{Q}} \mid v(\alpha)=1\right\}$ which has $\Gamma_{N}$ as a subgroup of finite index for some $N$;

$\left(2.8_{\mathrm{b}}\right)$ the inverse of the projection map coincides with $(2.7)$ on $\Gamma_{N}$ for sufficiently large $N$.

For such a $\Delta$ and a subfield $\mathfrak{R}$ of $\mathbf{C}$, we define $m_{\varrho, k}(\Delta, \mathfrak{H})\left(\right.$ resp. $\mathcal{A}_{\varrho, k}(\Delta, \Re)$ ) to be the set of all elements $f$ of $m_{Q, k}(\Re)$ (resp. $\mathcal{A}_{\ell, k}(\mathfrak{R})$ ) such that $f^{\beta}=f$ for all $\beta \in \Delta$. If $n>1,\left(2.8_{\mathrm{b}}\right)$ is always satisfied by virtue of the congruence subgroup property of $S p(n, \mathbf{Z})$.

Proposition 2.1. For every $k \in 2^{-1} \mathbf{Z},>0$, we have $m_{k}(\mathbf{C})=m_{k}(\mathbf{Q}) \otimes{ }_{\mathbf{Q}} \mathbf{C}$. Moreover, for each even positive integer $N$, let $\Delta_{N}$ be the image of $\Gamma_{N}$ under $(2.7)$; then $m_{k}\left(\Delta_{N}, \mathrm{C}\right)=$ $m_{k}\left(\Delta_{N}, \mathbf{Q}\right) \otimes \mathbf{Q} \mathbf{C}$.

Proof. As shown in $[5]$ and $[6, \S 3]$, there is a model $V$ defined over $Q$ for the compactification of $\mathfrak{S}_{n} / \Gamma_{N}$ whose function field can be identified with $\mathcal{A}_{0}\left(\Gamma_{N}, \mathbf{Q}\right)$; moreover, there 
is a non-zero element $g$ of $\mathcal{A}_{h}\left(\Gamma_{N}, \mathbf{Q}\right)$, for some positive integer $h$, whose divisor on $V$ is rational over $\mathbf{Q}$. We can take $h$ so that $\theta^{2 h} \in m_{h}\left(\Gamma_{2}, \mathbf{Q}\right)$. Then $\theta^{2 h} / g \in \mathcal{A}_{0}\left(\Gamma_{N}, \mathbf{Q}\right)$. It follows that the divisor of $\theta$ on $V$ is rational over $\mathbf{Q}$. Therefore, identifying $m_{k}\left(\Delta_{N}, \mathbf{Q}\right)$ with a linear system on $V$ rational over $\mathbf{Q}$ in the same manner as in the proof of [5, Th. 6], we obtain the second assertion, from which the first one follows immediately.

Proposition 2.2. The projection map of $(S)$ onto $G_{\mathbf{A}_{+}}$is open and continuous. Moreover, $(S)$ is locally compact, and $\iota_{1}\left(\mathbf{Z}_{\mathbf{f}}^{\times}\right) \mathscr{G}_{0} G_{\infty+}$ is dense in $\left(\mathcal{S}\right.$, where we embed $G_{\infty+}$ in (S) by identifying $x$ with $(x, 1)$ for $x \in G_{\infty+}$.

Proof. With an open compact subgroup $S$ of $G_{\mathrm{f}}$, a neighborhood $Y$ of the identity in $G_{\infty++}$, and a finite subset $F$ of $\mathcal{A}_{1 / 2}\left(\mathbf{Q}_{\mathrm{ab}}\right)$ containing $\theta$, we put

$$
U(S Y, F)=\left\{(x, v) \in \mathbb{S} \mid x \in S Y, h^{v}=h \quad \text { for all } h \in F\right\} .
$$

The sets of this type form a basis of neighborhoods of the identity element of 8 . Given such a set, let

$$
T=\left\{x \in S \mid(h / \theta)^{x}=h / \theta,\left(h^{2}\right)^{x}=h^{2} \quad \text { for all } h \in F\right\} .
$$

Then $T$ is an open compact subgroup of $G_{\mathrm{f}}$, and $U(S Y, F) \supset U(T Y, F)$. For every $x \in T Y$, define an element $v$ of $\&$ by $f^{v}=(f / \theta)^{x} \theta$. Then $(x, v) \in U(T Y, F)$. Thus we see that the projection of (S) onto $G_{\mathrm{A}+}$ gives a one-to-one map of $U(T Y, F)$ onto $T Y$. Our assertions except the last one follow from this fact. Let $(y, w)$ be an arbitrary element of Bs. Given $S$ and $F$, define $T$ as above, and put $y=x \iota(t) \alpha$ with $x \in T G_{\infty+}, t \in \mathbf{Z}_{\mathrm{f}}^{\times}$, and $\alpha \in G_{\mathbf{Q}+}$ by Lemma 1.1. Define again $v$ for $x$ as above. Then $(y, w)^{-1}(x, v) \iota_{1}(t)$ is an element of $\mathbb{S}$ whose projection to $G_{\mathrm{A}+}$ is $\alpha^{-1}$, and hence it belongs to $\mathbb{G}_{\mathbf{Q}}$. This proves the last assertion.

Given $F, S$, and $T$ as above, take an even positive integer $N$ so that $S_{N} \subset T G_{\infty}$. Obviously

$$
U\left(S_{N},\{\theta\}\right)=U\left(S_{N}, F\right) \subset U\left(T G_{\infty+}, F\right) .
$$

Thus the sets $U\left(S_{N},\{\theta\}\right)$ with $0<N \in \mathbf{Z}$ form a basis of neighborhoods of (S) modulo $G_{\infty++}$.

Proposition 2.3. For every even positive integer $N$, we have

$$
\begin{gathered}
U\left(S_{N},\{\theta\}\right) \cap \mathbb{S}_{\mathbf{Q}}=\Delta_{N}, \\
f^{y}=f \quad \text { if } y \in U\left(S_{N},\{\theta\}\right) \text { and } f \in \mathcal{A}_{Q, k}\left(\Delta_{N}, \mathbf{Q}(e(1 / N)) .\right.
\end{gathered}
$$

Proof. Equality (2.11) is obvious. To show (2.12), let $y \in U\left(S_{N},\{\theta\}\right), f \in \mathcal{A}_{Q, k}\left(\Delta_{N}\right.$, $Q(e(1 / N)))$, and $V=\left\{u \in U\left(S_{N},\{\theta\}\right) \mid\left(f / \theta^{k}\right)^{u}=f / \theta^{k}\right\}$. By Prop. 2.2, $y=u \iota_{1}(t) \beta$ with $u \in V$, $t \in \mathbf{Z}_{\mathbf{f}}^{\times}$and $\beta \in \mathfrak{S}_{\mathbf{Q}}$. We see that $t \equiv \mathbf{1}(\bmod N)$, and hence $\iota_{\mathbf{1}}(t) \in U\left(S_{N},\{\theta\}\right)$, so that $\beta \in \Delta_{N}$ by (2.11). Therefore $f^{y}=\left(f^{t}\right)^{\beta}=t$. 


\section{Generalization of theta functions}

In this section, we consider a certain class of holomorphic functions on $\mathbf{C}_{s}^{n} \times \mathscr{H}_{n}$ which includes classical theta functions as special cases, where $s$ and $n$ are arbitrary positive integers. First we define the action of an element $\alpha=\left(\begin{array}{ll}a & b \\ c & d\end{array}\right)$ of $G_{\mathbf{Q}+}$ on $\mathbf{C}_{s}^{n} \times \mathfrak{S}_{n}$ by

$$
\alpha(u, z)=\left({ }^{t}(c z+d)^{-1} u, \alpha(z)\right) \quad\left(u \in \mathbf{C}_{s}^{n}, z \in \mathfrak{S}_{n}\right) .
$$

We also define the action of an element $\beta=(\alpha, \psi)$ of $\mathfrak{S S}_{\mathbf{Q}}$ on $\mathfrak{S}_{n}$ and on $\mathbf{C}_{s}^{n} \times \mathfrak{S}_{n}$ to be the same as that of $\alpha$, and put $\nu(\beta)=\nu(\alpha)$. Throughout this section, $k$ will denote a non-negative element of $2^{-1} \mathbf{Z}$, and $\xi$ a non-negative symmetric element of $\mathbf{Q}_{s}^{s}$. Also we fix a polynomial representation

$$
\tau: G L_{n+s}(\mathbf{Q}) \rightarrow G L_{m}(\mathbf{Q}),
$$

and use the same notation $\tau$ for its natural extension to $G L_{n+s}(\mathbf{C})$. For a $\mathbf{C}^{m}$-valued function $f(u, z)$ on $\mathbf{C}_{s}^{n} \times \mathscr{\mathfrak { S }}_{n}$ and $\beta=(\alpha, \psi) \in \mathscr{G}_{\mathbf{Q}}$ with $\alpha=\left(\begin{array}{ll}a & b \\ c & d\end{array}\right)$, we define functions $\left.f\right|_{\tau, k} \beta$ and $\left.f\right|_{\tau, k, \xi} \beta$ on $\mathbf{C}_{s}^{n} \times \mathfrak{S}_{n}$ by

$$
\begin{gathered}
\left(\left.f\right|_{\tau_{,}, k} \beta\right)(u, z)=\psi(z)^{-2 k} \tau\left(\begin{array}{cc}
c z+d & 0 \\
0 & \nu(\alpha) 1_{s}
\end{array}\right)^{-1} f(\alpha(u, z)) \\
\left(\left.f\right|_{\tau, k, \xi} \beta\right)(u, z)=\psi(z)^{-2 k} e_{s}\left(-\frac{1}{2} \nu(\alpha)^{-1} \xi \cdot t u(c z+d)^{-1} c u\right) \tau\left(\begin{array}{cc}
c z+d & c u \\
0 & \nu(\alpha) 1_{s}
\end{array}\right)^{-1} f(\alpha(u, z)) .
\end{gathered}
$$

When $k \in \mathbf{Z}$ and $\psi(z)^{2}=\operatorname{det}(c z+d)$, we denote $\left.f\right|_{\tau, k} \beta$ and $\left.f\right|_{\tau, k, \xi} \beta$ also by $\left.f\right|_{\tau, k} \alpha$ and $\left.f\right|_{\tau, k, \xi} \alpha$.

Let us now associate with a given $f$ two $\mathbf{C}^{m}$-valued functions $P^{\tau, \xi} f$ and $P_{\tau, \xi} f$ defined by

$$
\begin{gathered}
\left(P^{\tau, \xi} f\right)(u, z)=e_{s}\left(\frac{1}{2} \xi \cdot t u(z-\bar{z})^{-1} u\right) \tau\left(\begin{array}{cc}
1_{n} & (z-\bar{z})^{-1} u \\
0 & 1_{s}
\end{array}\right) f(u, z), \\
\left(P_{\tau . \xi} f\right)(u, z)=e_{s}\left(\frac{1}{2} \xi \cdot t(u-\bar{u})(z-\bar{z})^{-1} u\right) \tau\left(\begin{array}{cc}
1_{n} & (z-\bar{z})^{-1}(u-\bar{u}) \\
0 & 1_{s}
\end{array}\right) f(u, z) .
\end{gathered}
$$

It can easily be verified that for every $\beta \in \mathfrak{B S}_{0}$,

$$
\begin{aligned}
& P^{\tau, \eta}\left(\left.f\right|_{\tau, k, \xi} \beta\right)=\left.\left(P^{\tau, \xi} f\right)\right|_{\tau, k} \beta, \\
& P_{\tau, \eta}\left(\left.f\right|_{\tau, k, \xi} \beta\right)=\left.\left(P_{\tau, \xi} f\right)\right|_{\tau, k} \beta \quad\left(\eta=v(\beta)^{-1} \xi\right)
\end{aligned}
$$

From this and an obvious relation $\left.f\right|_{\tau, k}(\beta \delta)=\left.\left(\left.f\right|_{\tau, k} \beta\right)\right|_{\tau, k} \delta$, we obtain

$$
\left.\left(\left.f\right|_{\tau, k, \xi} \beta\right)\right|_{\tau, k, \eta} \delta=\left.f\right|_{\tau, k, \xi}(\beta \delta) \quad \text { if } \eta=\boldsymbol{v}(\beta)^{-1} \xi
$$


Let $\Delta$ be a congruence subgroup of $\mathscr{G}_{\mathbf{Q}}$ as defined in $\S 2$, and $\Lambda$ a lattice in $\mathbf{Q}_{s}^{2 n}$. Our main object of study in this section is a holomorphic $\mathbf{C}^{m}$-valued function $f$ on $\mathbf{C}_{s}^{n} \times \mathfrak{Y}_{n}$ satisfying the following two conditions:

$$
\begin{gathered}
\left.f\right|_{\tau, k, \xi} \gamma=f \text { for all } \gamma \in \Delta, \\
f(u+z p+q, z)=e_{s}\left(-\xi\left(\frac{1}{2} \cdot{ }^{t} p z p+{ }^{t} p u\right)\right) \tau\left(\begin{array}{lr}
1_{n} & -p \\
0 & 1_{s}
\end{array}\right) f(u, z) \\
\text { for all }\left(\begin{array}{l}
p \\
q
\end{array}\right) \in \Lambda \text { with } p \text { and } q \text { in } \mathbf{Q}_{s}^{n} .
\end{gathered}
$$

Obviously such an $f$ is periodic as a function in the real parts of $u$ and $z$, so that it has a Fourier expansion of the form

$$
f(u, z)=\sum_{\eta \in Y} \sum_{\lambda \in L} c(\eta, \lambda) e_{n}(\eta z+u \lambda)
$$

with $c(\eta, \lambda) \in \mathbf{C}^{m}$, where $L$ is a lattice in $\mathbf{Q}_{n}^{s}$, and $Y$ is a lattice in the vector space $\left\{\left.\eta \in \mathbf{Q}_{n}^{n}\right|^{t} \eta=\eta\right\}$. If $n>1$, we denote by $T_{\tau, k, \xi}(\Delta, \Lambda)$ the vector space of satisfying (3.10) and (3.11). In the case $n=1$, we define $T_{\tau, k, \xi}(\Delta, \Lambda)$ under a certain additional condition (3.23) below. For the moment, let us assume either $n>1$, or as if the definition for $n=1$ is already given.

For a subfield $\Re$ of $\mathrm{C}$, we denote by $T_{\tau, k, \xi}(\Delta, \Lambda ; \Re)$ the set of all $f \in T_{\tau, k, \xi}(\Delta, \Lambda)$ with expansion (3.12) whose coefficients $c(\eta, \lambda)$ have components in $\Re$. Further we denote by $T_{\tau, k, \xi}(\Re)$ the union of $T_{\tau, k, \xi}(\Delta, \Lambda ; \Re)$ for all possible $\Delta$ and $\Lambda$, and put $T_{\tau, k, \xi}=T_{\tau, k, \xi}(\mathbf{C})$. If $k \in \mathbf{Z}, T_{\tau, k, \xi}(\Gamma, \Lambda)$ and $T_{\tau, k, \xi}(\Gamma, \Lambda ; \Re)$ can be defined for a congruencesubgroup $\Gamma$ of $G_{\mathbf{O}+}$ in a similar way. To simplify our notation, we write hereafter $f^{*}, f_{*}$, and $f^{\beta}$ for $P^{\tau, \xi} f$, $P_{\tau, \xi} f$, and $\left.f\right|_{\tau, k, \xi} \beta$, when $f \in T_{\tau, k, \xi}$ or more generally when $f$ satisfies $(3.10)$ and ${ }_{-}^{7}(3.11)$; further, if $\tau$ is trivial we write $T_{k, \xi}$ for $T_{\tau, k, \xi}$.

For each $z \in \mathfrak{S}_{n}$, define a hermitian form $H_{\xi, z}$ and an alternating form $E_{\xi, z}$ by

$$
\begin{aligned}
H_{\xi, z}(u, v) & =2 i \cdot \operatorname{tr}\left(\xi \cdot{ }^{t} \bar{u}(z-\bar{z})^{-1} v\right), \\
2 i \cdot E_{\xi, z}(u, v) & =H_{\xi, z}(u, v)-H_{\xi, z}(v, u) \quad\left(u, v \in \mathbf{C}_{s}^{n}\right) .
\end{aligned}
$$

Put $\Omega_{z}=\left(\begin{array}{ll}z & 1_{n}\end{array}\right)$. Then

$$
E_{\xi, z}\left(\Omega_{z} a, \Omega_{z} b\right)=\operatorname{tr}(\xi \cdot t a J b) \quad\left(a, b \in \mathbf{R}_{s}^{2 n}\right) .
$$

If $f \in T_{\tau_{\star} k, \xi}(\Delta, \Lambda)$ and $l=z p+q$ with $\left(\begin{array}{l}p \\ q\end{array}\right) \in \Lambda$, we have

$$
\begin{gathered}
f^{*}(u+l, z)=e_{s}\left(\frac{1}{2} \xi \cdot{ }^{t} p q\right) e\left(\frac{1}{2 i} H_{\xi, z}\left(l, u+\frac{1}{2} l\right)\right) \tau\left(\begin{array}{cc}
1_{n} & (z-\bar{z})^{-1} \bar{l} \\
0 & 1_{s}
\end{array}\right) f^{*}(u, z) \\
f_{*}(u+l, z)=e_{s}\left(\frac{1}{2} \xi \cdot{ }^{t} p q\right) e\left(E_{\xi, z}(l, u) / 2\right) f_{*}(u, z) .
\end{gathered}
$$


Now, for $f \in T_{\tau, k, \xi}$ and elements $r$ and $r^{\prime}$ of $\mathbf{R}_{s}^{n}$, we have

$$
f_{*}\left(z r+r^{\prime}, z\right)=e_{s}\left(\frac{1}{2} \xi \cdot{ }^{t} r\left(z r+r^{\prime}\right)\right) \tau\left(\begin{array}{ll}
1_{n} & r \\
0 & 1_{s}
\end{array}\right) f\left(z r+r^{\prime}, z\right)
$$

This is obviously holomorphic in $z$. Now for $\beta=(\alpha, \psi) \in G_{\mathbf{Q}}$ with $\alpha=\left(\begin{array}{ll}a & b \\ c & d\end{array}\right)$ and $v \in \mathbf{R}_{s}^{2 n}$, we have

$$
f_{*}\left(\Omega_{\alpha(z)} v, \alpha(z)\right)=\psi(z)^{2 k} \tau\left(\begin{array}{cc}
c z+d & 0 \\
0 & v(\alpha) 1_{s}
\end{array}\right)\left(f^{\beta}\right)_{*}\left(\Omega_{z} \cdot{ }^{t} \alpha v, z\right)
$$

This follows immediately from (3.8). Therefore, if we define a representation $\varrho$ of $G L_{n}$ by

$$
\varrho(X)=\tau\left(\begin{array}{ll}
X & 0 \\
0 & 1_{s}
\end{array}\right)
$$

and put $h_{v}(z)=f_{*}\left(\Omega_{z} v, z\right)$ and $h_{v}^{\prime}(z)=\left(f^{\beta}\right)_{*}\left(\Omega_{z} v, z\right)$, then

$$
\left.h_{v}\right|_{e, k} \beta=\tau\left(\begin{array}{cc}
\mathbf{1}_{n} & 0 \\
0 & \nu(\alpha) \mathbf{l}_{s}
\end{array}\right) h_{w}^{\prime} \text {, where } w={ }^{t} \alpha v
$$

On the other hand, put $g(v)=f_{*}\left(\Omega_{z} v, z\right)$ for $v \in \mathbf{Q}_{s}^{2 n}$ with a fixed $z$. Then (3.17) together with (3.15) shows that, given any lattice $L$ in $\mathbf{Q}_{s}^{2 n}$, we can find another lattice $L^{\prime} \subset L$ so that $g$ defines a function on $L / L^{\prime}$. Therefore, if $\mathbf{A}_{\mathrm{f}}$ denotes the non-archimedean part of $\mathbf{A}$, then $g$ can be uniquely extended to a continuous function on $\left(\mathbf{A}_{\mathbf{f}}\right)_{s}^{2 n}$. (Cf. [7, pp. 683-4].) Thus $f_{*}\left(\Omega_{z} v, z\right)$ for $v \in\left(\mathbf{A}_{\mathbf{f}}\right)_{s}^{2 n}$ is meaningful. Coming back to the above $h_{v},(3.21)$ implies

(3.22) $\left.h_{v}\right|_{\ell . k} \gamma=h_{v}$ if $v \in \mathbf{Q}_{s}^{2 n}$ and $\gamma$ belongs to a sufficiently small congruence subgroup of $\mathfrak{S}_{\mathbf{Q}}$ depending on $v$.

Therefore if $n>1$, we see that

(3.23) $f_{*}\left(\Omega_{z} v, z\right)$ for every fixed $v \in \mathbf{Q}_{s}^{2 n}$ belongs, as a function of $z$, to $m_{Q, k}(\mathbf{C})$, where $\varrho$ is defined by (3.20).

Now in the case $n=1$, we define $T_{\tau, k, \xi}(\Delta, \Lambda)$ to be the set of all holomorphic $f$ satisfying (3.10), (3.11), and (3.23). Condition (3.23) is essentially a condition on the Fourier coefficients of $f$ (at all cusps) as shown by the following proposition and its proof.

Proposition 3.1. If $f$ is an element of $T_{\tau, k, \xi}$ with expansion $(3.12)$, then $c(\eta, \lambda) \neq 0$ only when $\eta \geqslant 0$, and moreover, for a fixed $\eta$, there are only finitely many $\lambda$ such that $c(\eta, \lambda) \neq 0$. 
Proof. Observe that if $p, q \in \mathbf{Q}_{s}^{n}$, we have

with

$$
f_{*}(z p+q, z)=\tau\left(\begin{array}{ll}
1_{n} & p \\
0 & 1_{s}
\end{array}\right) \sum_{\zeta} a(\zeta) e_{n}(\zeta z)
$$

$$
a(\zeta)=e_{s}\left(\xi \cdot{ }^{t} p q / 2\right) \sum_{\lambda_{n} \eta} e_{s}(\lambda q) c(\eta, \lambda)
$$

where the sum is taken under the condition

$$
2 \zeta=2 \eta+p \xi \cdot{ }^{t} p+p \lambda+{ }^{t} \lambda \cdot{ }^{t} p .
$$

If $\zeta$ is not non-negative, (3.23) implies that (3.24) must be 0 for all $q \in \mathbf{Q}_{s}^{n}$. Therefore

$$
c(\eta, \lambda) \neq 0 \quad \text { only if } 2 \eta+p \xi \cdot{ }^{t} p+p \lambda+{ }^{t} \lambda \cdot{ }^{t} p \geqslant 0 \quad \text { for all } p \in \mathbf{Q}_{s}^{n} .
$$

Taking $p$ to be 0 , we see that $c(\eta, \lambda)=0$ unless $\eta \geqslant 0$. For a fixed $\eta$, there are only finitely many $\lambda$ in a given lattice satisfying the inequality of (3.25) for all $p \in \mathbf{Q}_{s}^{n}$. This completes the proof.

Note that the sum of (3.24) is a finite sum. In fact, if $c(\eta, \lambda) \neq 0$, we have $2 \eta+4 p \xi \cdot{ }^{t} p+$ $2 p \lambda+2 \cdot{ }^{t} \lambda \cdot{ }^{t} p \geqslant 0$ by $(3.25)$, so that $\left(3.24^{\prime}\right)$ shows $\eta \leqslant 2 \zeta+p \xi \cdot{ }^{t} p$. There are only finitely many such $\eta \geqslant 0$ in a lattice for fixed $p$ and $\zeta$, which proves the desired finiteness.

Propositron 3.2. Let $\Re$ be a subfield of $\mathbf{C}$ containing $\mathbf{Q}_{\mathrm{ab}}$, and let $f \in T_{\tau, k, \xi}$. Then (i) $f \in T_{\tau, k, \xi}(\Re)$ if and only if $f_{*}\left(\Omega_{z} v, z\right) \in M_{Q, k}(\Re)$ for all $v \in \mathbf{Q}_{s}^{2 n}$; (ii) if $f \in T_{\tau, k, \xi}(\Re)$ and $\beta \in \mathscr{G}_{\mathbf{Q}}$, then $f^{\beta} \in T_{\tau, k, \zeta}(\Re)$ with $\zeta=v(\beta)^{-1} \xi$.

Proof. Consider expansion (3.12) for $f$. If $f \in T_{\tau, k, \xi}(\Re)$, then obviously $f_{*}\left(\Omega_{z} v, z\right) \in$ $m_{e, k}(\mathfrak{R})$ for all $v \in \mathbf{Q}_{s}^{2 n}$. Conversely suppose $f_{*}(r, z)$ has Fourier coefficients in $\mathfrak{h}^{m}$ for every $r \in \mathbf{Q}_{s}^{n}$. This implies that

$$
\sum_{\lambda} c(\eta, \lambda) e_{s}(\lambda r) \in \Re^{m} \quad \text { for all } r \in \mathbf{Q}_{s}^{n},
$$

and hence $c(\eta, \lambda) \in \Re^{m}$. To prove assertion (ii), first assume $\mathfrak{R}=\mathbf{C}$. If $n>1$, the desired conclusion follows easily from our definition of $T_{\tau, k, \xi}$; if $n=1$, we need (3.21). This result together with assertion (i), (3.21), and the fact that $m_{\varrho, k}(\Re)^{\beta}=m_{\varrho, k}(\Re)$ proves assertion (ii) in the general case.

Typical examples of functions of $T_{k, \xi}$ with $s=1$ are provided by

$$
\begin{gathered}
\theta(u, z ; p, q)=\sum_{x-p \in \mathbf{Z}^{n}} e\left(\frac{1}{2} \cdot{ }^{t} x z x+{ }^{t} x(u+q)\right), \\
\varphi(u, z ; p, q)=e\left(\frac{1}{2} \cdot{ }^{t} u(z-\bar{z})^{-1} u\right) \theta(u, z ; p, q), \\
\varphi^{\prime}(u, z ; p, q)=e\left(\frac{1}{2} \cdot{ }^{t}(u-\bar{u})(z-\bar{z})^{-1} u\right) \theta(u, z ; p, q),
\end{gathered}
$$

4-782901 Acta mathematica 141. Imprimé le 1 Septembre 1978 
where $u \in \mathbf{C}^{n}$ and $p, q \in \mathbf{R}^{n}$. In fact, if $f(u, z)=\theta(\lambda u, \mu z ; p, q)$ with $p, q \in \mathbf{Q}^{n}$ and positive rational numbers $\lambda, \mu$, then $f \in T_{1 / 2, v}\left(\mathbf{Q}_{\mathrm{ab}}\right)$ with $\nu=\lambda^{2} / \mu$, and

$$
f^{*}(u, z)=\varphi(\lambda u, \mu z ; p, q), \quad f_{*}(u, z)=\varphi^{\prime}(\lambda u, \mu z ; p, q)
$$

(cf. Appendix and $[7, \S 1])$. To give an explicit example of $(3.21)$ or $(3.22)$, put

$$
\begin{aligned}
\chi(z ; v, w ; p, q) & =\varphi^{\prime}(z v+w, z ; p, q) & & \left(v, w, p, q \in \mathbf{R}^{n}\right), \\
\psi_{\gamma} & =(\theta \circ \gamma) / \theta & & \left(\gamma \in \Gamma_{\theta}\right) .
\end{aligned}
$$

We obtain, from (a.3) of the Appendix,

$$
\chi(\gamma(z) ; v, w ; p, q)=e\left(\left({ }^{t} p q-{ }^{t} p^{*} q^{*}\right) / 2\right) \psi_{\gamma}(z) \chi\left(z ; v^{*}, w^{*} ; p^{*}, q^{*}\right)
$$

for every $\gamma \in \Gamma_{\theta}$, where

$$
\left(\begin{array}{c}
v^{*} \\
w^{*}
\end{array}\right)={ }^{t} \gamma\left(\begin{array}{c}
v \\
w
\end{array}\right),\left(\begin{array}{l}
p^{*} \\
q^{*}
\end{array}\right)={ }^{t} \gamma\left(\begin{array}{l}
p \\
q
\end{array}\right)
$$

Also we note that

$$
\chi(z ; v, w ; p, q)=e\left(-\frac{1}{2} \cdot{ }^{t} v w-{ }^{t} v q\right) \theta(0, z ; v+p, w+q)
$$

This follows from $[7,(11)]$ and was actually stated as $[7,(27)]$.

For $f \in T_{\tau, k, \xi}(\Delta, \Lambda ; \Re)$ and $\varepsilon \in G L_{s}(\mathbf{Q})$, put

$$
g(u, z)=\tau\left(\begin{array}{ll}
\mathbf{l}_{n} & 0 \\
0 & \varepsilon
\end{array}\right) f(u \varepsilon, z), \zeta=\varepsilon \xi \cdot{ }^{t} \varepsilon .
$$

Then $g \in T_{\tau, k, \zeta}\left(\Delta, \Lambda \varepsilon^{-1} ; \Re\right)$. Therefore, to discuss the nature of $f$, we can simplify our discussion by assuming that

$$
\xi=\operatorname{diag}\left[\xi_{1}, \ldots, \xi_{r}, 0, \ldots, 0\right], \quad r=\operatorname{rank}(\xi),
$$

with positive integers $\xi_{i}$, and also that $\Lambda \supset \mathbf{Z}_{s}^{2 n}$.

Proposition 3.3. Suppose that $\xi$ is given by (3.32), and write the variable $u$ on $\mathbf{C}_{s}^{n}$ in the form $u=(v, w)$ with $v \in \mathbf{C}_{r}^{n}$ and $w \in \mathbf{C}_{s-r}^{n}$. Define a representation $\omega$ of $G L_{n+}$, by

$$
\omega(X)=\tau\left(\begin{array}{ll}
X & 0 \\
0 & 1_{s-r}
\end{array}\right) \quad\left(X \in G L_{n+r}\right)
$$

Then, for each $f \in T_{\tau, k, \xi}$, there is an element $g$ of $T_{\omega, k, \eta}$ such that $f(v, w, z)=g(v, z)$, where $\eta=\operatorname{diag}\left[\xi_{1}, \ldots, \xi_{r}\right]$. Moreover, $f_{*}(v, w, z)=g_{*}(v, z)$ and

$$
f^{\beta}(v, w, z)=\tau\left(\begin{array}{lc}
\mathbf{1}_{n+r} & 0 \\
0 & \nu(\beta)^{-1} 1_{s-r}
\end{array}\right) g^{\beta}(\bar{v}, z) \text { for every } \beta \in \mathcal{S}_{\mathbf{Q}} .
$$


Proof. For any fixed $v$ and $z$, let $h(w)$ be a component of the vector $f_{*}(v, w, z)$. Then (3.17) implies that

$$
h(w+z p+q)=h(w) \text { for all } p, q \in L,
$$

where $L$ is a lattice in $\mathbf{Q}_{s-r}^{n}$. Now (3.6) shows that $h$ is a finite $\operatorname{sum} \sum_{\nu} A_{\nu}(w) B_{\nu}(x)$ with holomorphic functions $A_{\nu}$ and monomials $B_{\nu}$ of the components, say $x_{i j}$, of $x=\operatorname{Im}(w)$. Let us consider this as a polynomial in $x_{i j}$, and prove, by induction on the degree of the polynomial, that it is a constant, involving neither $w$ nor $x$. First suppose that it is of degree 0 , i.e., $h$ is holomorphic in $w$. Then (3.33) implies that $h$ is a holomorphic function on a complex torus, which must be a constant. In the general case, applying the induction assumption to $\partial h / \partial \bar{w}_{i j}$, we see that $h(w)=A_{0}(w)+\sum_{i, j} a_{i j} \bar{w}_{i j}$ with a holomorphic $A_{0}$ and $a_{i j} \in \mathbf{C}$. Then (3.33) shows that $\partial A_{0} / \partial w_{i j}$ is invariant under $w \mapsto w+z p+q$, and hence is a constant. It follows that $h(w)=b+\operatorname{tr}(D w+E \bar{w})$ with $b \in \mathbf{C}$ and $D, E \in C_{n}^{s-r}$. Again from (3.33), we obtain

$$
\operatorname{tr}(D(z p+q)+E(\bar{z} p+q))=0 \text { for all } p, q \in L,
$$

so that $D=E=0$. Thus we have proved that $f_{*}(v, w, z)$ does not depend on $w$. This together with (3.6) shows that $f(v, w, z)$, for fixed $v$ and $z$, is a polynomial function in $w-\bar{w}$. Being holomorphic in $w$, it must be a constant. Thus we can put $f(v, w, z)=g(v, z)$. Then it is straightforward to verify all our assertions.

Before proceeding further, we prove an easy

LEMMA 3.4. Let $A$ be an arbitrary set, and $B$ a domain in $\mathbf{C}^{n}$, and let $f_{1}(u, z), \ldots, f_{m}(u, z)$, $g(u, z)$ be complex valued functions on $A \times B$, holomorphic in $z$, where $u \in A$ and $z \in B$. Suppose that the functions $f_{1}\left(u, z_{0}\right), \ldots, f_{m}\left(u, z_{0}\right)$ on $A$ are linearly independent over $\mathbf{C}$ for every $z_{0} \in B$, and $g(u, z)=\sum_{k=1}^{m} h_{k}(z) f_{k}(u, z)$ with functions $h_{k}$ on B. Then $h_{k}$ is holomorphic on B for every $k$.

Proof. For fixed $u$ and $z$, put

$$
X(u, z)=\left\{\left(c_{\mathbf{1}}, \ldots, c_{m}\right) \in \mathbf{C}_{m}^{1} \mid \sum_{k} c_{k} f_{k}(u, z)=0\right\} .
$$

For any fixed $z_{0} \in B$, we have $\bigcap_{u \in A} X\left(u, z_{0}\right)=\{0\}$, so that there exist $m$ points $u_{1}, \ldots, u_{m}$ of $A$ such that $\bigcap_{j=1}^{m} X\left(u_{j}, z_{0}\right)=\{0\}$. This means that $\operatorname{det}\left(f_{k}\left(u_{j}, z_{0}\right)\right)_{j, k} \neq 0$. Solving the equations

$$
g\left(u_{j}, z\right)=\sum_{k=1}^{m} h_{k}(z) f_{k}\left(u_{n}, z\right) \quad(j=1, \ldots, m)
$$

we see that $h_{k}$ is holomorphic in a neighborhood of $z_{0}$, QED. 
Proposition 3.5. If $n>1$, every $f$ of $T_{k, \xi}(\Re)$ has an expression

$$
f(u, z)=\sum_{h \in H / K} c_{h}(z) \sum_{x-h \in K} e_{s}\left(\xi\left(\frac{1}{2} \cdot{ }^{t} x z x+{ }^{t} x u\right)\right)
$$

with $c_{h} \in m_{k-(r / 2)}(\Re)$, where $H$ and $K$ are lattices in the vector space

$$
\mathbf{Q}_{s}^{n} /\left\{y \in \mathbf{Q}_{s}^{n} \mid y \xi=0\right\}
$$

and $r=\operatorname{rank}(\xi)$. Conversely, any such expression defines an element of $T_{k, \xi}(\Re)$; this is so even if $n=1$.

Proof. Suppose $f \in T_{k, \xi}(\Delta, \Lambda)$. Take $\varepsilon \in G L_{s}(\mathbf{Q})$ so that $\mathbf{Z}_{s}^{2 n} \subset \Lambda \varepsilon^{-1}$ and $\varepsilon \xi \cdot{ }^{t} \varepsilon=\operatorname{diag}\left[\zeta_{1}, \ldots\right.$, $\left.\zeta_{r}, 0, \ldots, 0\right]$ with positive integers $\zeta_{\nu}$. Put $\zeta=\varepsilon \xi \cdot{ }^{t} \varepsilon$ and $g(u, z)=f(u \varepsilon, z)$. Then $g \in T_{k, \zeta}\left(\Delta, \mathbf{Z}_{s}^{2 n}\right)$. Let $u_{v}$ be the $v$-th column of $u$. By Prop. 3.3,g depends only on $u_{1}, \ldots, u_{r}$, and if $v \leqslant r$, we have

$$
g\left(\ldots, u_{\nu}+z p+q, \ldots, z\right)=e\left(-\zeta_{\nu}\left(\frac{1}{2} \cdot{ }^{t} p z p+{ }^{t} p u_{\nu}\right)\right) g\left(\ldots, u_{\nu}, \ldots, z\right)
$$

for $p, q \in \mathbf{Z}^{n}$. Therefore, as a function of $u_{\nu}$, it is a linear combination of $\theta\left(\zeta_{\nu} u_{\nu}, \zeta_{\nu} z ; h, 0\right)$ with $h$ in a set of representatives for $\zeta_{v}^{-1} \mathbf{Z}^{n} / \mathbf{Z}^{n}$. Consequently we obtain, in view of Lemma 3.4 ,

$$
\begin{aligned}
g(u, z) & =\sum_{h} c_{h}(z) \prod_{\nu=1}^{r} \theta\left(\zeta_{\nu} u_{v}, \zeta_{\nu} z ; h_{v}, 0\right) \quad\left(h=\left(h_{1}, \ldots, h_{r}\right)\right) \\
& =\sum_{h \in M / L} c_{h}(z) \sum_{x-h \in L} e_{r}\left(\zeta^{\prime}\left(\frac{1}{2} \cdot{ }^{t} x z x+{ }^{t} x u^{\prime}\right)\right)
\end{aligned}
$$

with holomorphic functions $c_{h}$ on $\mathfrak{W}_{n}$, where $\zeta^{\prime}=\operatorname{diag}\left[\zeta_{1}, \ldots, \zeta_{r}\right], u^{\prime}=\left(u_{1}, \ldots, u_{r}\right), L=\mathbf{Z}_{r}^{n}$, and $M=\mathbf{Z}_{r}^{n} \zeta^{\prime-1}$. Since $g$ satisfies (3.10), we can easily verify that $c_{n} \in m_{k-(r / 2)}(\Re)$ if $n>1$. Consider $L$ and $M$ as submodules of $\mathbf{Q}_{s}^{n}$ in an obvious way, and let $H$ and $K$ be their images under $\varepsilon$. Then we obtain our first assertion. The converse part follows immediately from the fact that $\theta(\lambda u, \mu z ; h, 0)$ defines an element of $T_{1 / 2, \nu}(Q)$ with $\nu=\lambda^{2} / \mu$.

LEMм A 3.6. For $f \in T_{k, \xi}(\Re)$, put

$$
d_{\lambda} f=\left[\begin{array}{cc}
\frac{1}{2 \pi i} \frac{\partial f}{\partial u_{1 \lambda}} \\
\vdots \\
\frac{1}{2 \pi i} \frac{\partial f}{\partial u_{n \lambda}} \\
\xi_{1 \lambda} f \\
\vdots \\
\xi_{s \lambda} f
\end{array}\right] \quad(\lambda=1, \ldots, s)
$$


Then $d_{\lambda} f \in T_{\tau, k, \xi}(\Re)$ with $\tau(X)=X$, and

$$
d_{\lambda}\left(f^{\beta}\right)=\left(\begin{array}{cc}
1_{n} & 0 \\
0 & \nu(\beta) 1_{s}
\end{array}\right)\left(d_{\lambda} f\right)^{\beta} \text { for every } \beta \in \mathscr{S}_{\mathbf{Q}} .
$$

This can be verified in a straightforward way, except the fact that $d_{\lambda}(f)$ satisfies (3.23) when $n=1$, which can be shown as follows. Since $f^{\beta}$ satisfies (3.25) for every $\beta \in \mathfrak{G S}_{\mathbf{Q}}$, we see easily that $d_{\lambda}\left(f^{\beta}\right)$ satisfies the same condition. Therefore, by $\left(3.36^{\prime}\right)$ and $(3.2 \mathrm{I})$, $\left(d_{\lambda} f\right)_{*}\left(\Omega_{z} v, z\right)^{\beta}$ is finite at $i \infty$ for every $v \in \mathbf{Q}_{s}^{2}$ and every $\beta \in \mathscr{S}_{\mathbf{Q}}$. Q.E.D.

Proposition 3.7. Suppose that $\xi$ is non-degenerate. Then, for an arbitrary point $\left(u_{0}, z_{0}\right)$ of $\mathbf{C}_{s}^{n} \times \mathfrak{H}_{n}$, there exists a function $A$ on $\mathbf{C}_{s}^{n} \times \mathfrak{H}_{n}$ with values in $\mathbf{C}_{m}^{m}$ such that

(i) the columns of $A$ belong to $T_{\tau, j, \xi}(\mathbf{Q})$, where $j$ is a positive element of $2^{-1} \mathbf{Z}$ depending only on $\tau$;

(ii) $\operatorname{det}\left(A\left(u_{0}, z_{0}\right)\right) \neq 0$.

Proof. If $\tau$ is trivial, the function $f$ defined by

$$
f(u \varepsilon, z)=c(z) \prod_{v=1}^{s} \theta\left(\zeta_{v} u_{v}, \zeta_{v} z ; h_{v}, 0\right)
$$

with suitable $c \in M_{j-(s / 2)}(Q)$ and $h_{\nu}$ can be taken as $A$, where $\varepsilon$ and $\zeta_{\nu}$ are as in the proof of Prop. 3.5. In this case $j$ may be arbitrarily chosen under the condition $j \geqslant s / 2$. Next assume $\tau(X)=X$. Let $k \geqslant s / 2$ and take $f \in T_{k, \xi}(Q)$ so that $f\left(u_{0}, z_{0}\right) \neq 0$. We are going to define $A$ by

$$
A(u, z)=\left[\begin{array}{ccccccc}
\frac{1}{2 \pi i} \frac{\partial f}{\partial u_{11}} & \ldots & \frac{1}{2 \pi i} \frac{\partial f}{\partial u_{1 s}} b_{11} & \ldots & b_{1 n} \\
\ldots & \ldots & \ldots & \ldots & \ldots & \ldots \\
\frac{1}{2 \pi i} \frac{\partial f}{\partial u_{n 1}} & \ldots & \frac{1}{2 \pi i} \frac{\partial f}{\partial u_{n s}} & b_{n 1} & \ldots & b_{n n} \\
\xi_{11} f & \ldots & \xi_{1 s} f & 0 & \ldots & 0 \\
\ldots & \ldots & \ldots & \ldots & \ldots & \ldots \\
\xi_{s 1} f & \ldots & \xi_{s s} f & 0 & \ldots & 0
\end{array}\right]
$$

with a suitable $B=\left(b_{\lambda \mu}(u, z)\right)$. Let $\varrho$ be the identity representation of $G L_{n}(\mathbf{C})$ onto itself. By [8, Prop. 1.2] and its proof, we can find an element $P$ of $m_{\varrho, 1 / 2}(Q)$ such that $\operatorname{det}\left(P\left(z_{0}\right)\right) \neq$ 0 . Take $g \in T_{k-(1 / 2), \xi}(\mathbf{Q})$ so that $g\left(u_{0}, z_{0}\right) \neq 0$, and put $B(u, z)=g(u, z) P(z)$. By virtue of Lemma 3.6, $A$ has the required property. In this case, $j$ must be $>s / 2$. Let $\omega(X)=X \otimes \ldots$ $\otimes X$ and $C=A \otimes \ldots \otimes A$ (both $t$ copies). Then $\operatorname{det}\left(C\left(u_{0}, z_{0}\right)\right) \neq 0$, and the columns of $C$ belong to $T_{\omega, t t_{*} t \xi}(\mathbf{Q})$. Since an arbitrary irreducible $\tau$ is a Q-rational component of such $\omega$, we obtain our assertion. 
Propositron 3.8. $T_{\tau, k, \xi}=T_{\tau, k, \xi}(\mathbf{Q}) \otimes \mathbf{Q} \mathrm{C}$.

This will be proved in the next section.

We are going to define an action of $\mathbb{S}$ on $T_{\tau, k, \xi}\left(\mathbf{Q}_{\mathrm{ab}}\right)$. First, for $f \in T_{\tau, k, \xi}(\Re)$ with expansion (3.12) and an injection $\sigma$ of $\Re$ into $\mathrm{C}$, we define $f^{\sigma}$ by

$$
f^{\sigma}(u, z)=\sum_{\eta, \lambda} c(\eta, \lambda)^{\sigma} e_{n}(\eta z+u \lambda)
$$

By virtue of Prop. 3.8, $f^{\sigma}$ actually defines an element of $T_{\tau, k, \xi}$. In particular, if $f \in T_{\tau, k, \xi}\left(\mathbf{Q}_{\mathrm{ab}}\right), f^{b}$ is meaningful for $b \in \mathbf{A}^{\times}$.

As already shown, $f_{*}\left(\Omega_{z} v, z\right)$ is meaningful for $v \in\left(\mathbf{A}_{\mathbf{f}}\right)_{s}^{2 n}$, and defines, as a function of $z$, an element of $m_{Q, k}(\mathbf{C})$, where $\varrho$ is given by $(3.20)$. Now we let $G_{\mathbf{A}}$ act on $\left(\mathbf{A}_{\mathbf{i}}\right)_{s}^{2 n}$ by left matrix multiplication, of course ignoring the archimedean part.

Proposition 3.9. Let $f \in T_{\tau, k, \xi}, v \in\left(\mathbf{A}_{\mathfrak{f}}\right)_{s}^{2 n}, r \in \mathbf{Z}_{\mathfrak{f}}^{\times}$, and let $\sigma$ be an automorphism of $\mathbf{C}$ that coincides with the action of $r$ on $\mathbf{Q}_{\mathrm{ab}}$. Then

$$
f_{*}\left(\Omega_{z} v, z\right)^{\sigma}=\left(f^{\sigma}\right)_{*}\left(\Omega_{z} \iota(r) v, z\right)
$$

where the left-hand side is defined by the action of $\sigma$ on $m_{0, k}(\mathbf{C})$.

Proof. If $v=\left(\begin{array}{l}p \\ q\end{array}\right)$ with $p$ and $q$ in $\mathbf{Q}_{s}^{n}$, and if $f$ has expansion (3.12), then, as shown in the proof of Prop. 3.1, we have

$$
f_{*}\left(\Omega_{z} v, z\right)=\tau\left(\begin{array}{ll}
1_{n} & p \\
0 & 1_{s}
\end{array}\right) \sum_{\zeta} a(\zeta) e_{n}(\zeta z)
$$

with $a(\zeta)$ given by (3.24). Since the sum expressing $a(\zeta)$ is a finite sum, we have

$$
a(\zeta)^{\sigma}=e_{s}\left(\xi \cdot{ }^{t} p q^{\prime} / 2\right) \sum_{\lambda} e_{s}\left(\lambda q^{\prime}\right) c(\eta, \lambda)^{\sigma}
$$

where $q^{\prime}$ is an element of $\mathbf{Q}_{s}^{n}$ sufficiently close to $r^{-1} q$. This proves our proposition.

We are now ready to state our first main result:

TH EOR EM 3.10. Given $f \in T_{\tau, k, \xi}\left(\mathbf{Q}_{\mathrm{ab}}\right)$ and $y \in(G)$, there is a unique element $f^{y}$ of $T_{\tau, k, \eta}\left(\mathbf{Q}_{\mathrm{ab}}\right)$ with $\eta=|v(x)|^{-1} \xi$ such that

$$
f_{*}\left(\Omega_{z} v, z\right)^{y}=\tau\left(\begin{array}{cc}
1_{n} & 0 \\
0 & |v(x)| 1_{s}
\end{array}\right)\left(f^{y}\right)_{*}\left(\Omega_{z} \cdot{ }^{t} x v, z\right) \text { for all } v \in\left(\mathbf{A}_{\mathbf{f}}\right)_{s}^{2 n},
$$

where $x$ is the projection of $y$ to $G_{\mathbf{A}_{+}}$, and $f_{*}\left(\Omega_{z} v, z\right)^{y}$ is the image of $f_{*}\left(\Omega_{z} v, z\right)$ as an element of $M_{Q, k}\left(Q_{\mathrm{ab}}\right)$ under $y$ as defined in $\S 2$. If $k \in \mathbf{Z}$, the assertion holds with $G_{\mathrm{A}+}$ and $x$ in place of (S) and $y$. 
Proof. Define $\Delta_{N}$ as in Prop. 2.1. In view of Prop. 3.8, we can find a positive integer $N$ and a lattice $\Lambda$ such that $f^{t} \in T_{\tau, k, \xi}\left(\Delta_{N}, \Lambda ; \mathbf{Q}(e(1 / N))\right)$ for all $t \in \mathbf{Z}_{\mathrm{f}}^{\times}$. Define $U\left(S_{N},\{\theta\}\right)$ by (2.9). By Prop. 2.2, we have $y=y^{\prime} \iota_{\mathbf{1}}(t) \beta$ with $y^{\prime} \in U\left(S_{N},\{\theta\}\right), t \in \mathbf{Z}_{\mathfrak{f}}^{\times}$and $\beta \in \mathscr{S S}_{\mathbf{Q}}$. Now we define $f^{y}$ to be $\left(f^{t}\right)^{\beta}$. By our choice of $N$, we see easily that this is independent of the choice of $y^{\prime}, t, \beta$, in view of Prop. 2.3. Moreover, $f^{y} \in T_{\tau, k, \eta}\left(\mathbf{Q}_{\mathrm{ab}}\right)$ by Prop. 3.2, since $v(\beta)=$ $|v(x)|$. To show (3.38), given $v \in \mathbf{Q}_{s}^{2 n}$, take a multiple $M$ of $N$ so that $M v \in \mathbf{Z}_{s}^{2 n}$ and $f_{*}\left(\Omega_{z} v, z\right) \in$ $m_{\varrho, k}\left(\Delta_{M}, \mathbf{Q}(e(1 / M))\right)$. Changing $y^{\prime}, t$, and $\beta$ if necessary, we may assume that $y^{\prime} \in U\left(S_{M},\{\theta\}\right)$. By Prop. 2.3, (2.5), (2.6), (3.19), and Prop. 3.9, we have

$$
\begin{aligned}
f_{*}\left(\Omega_{z} v, z\right)^{y} & =\left(f_{*}\left(\Omega_{z} v, z\right)^{t}\right)^{\beta}=\left(f^{t}\right)_{*}\left(\Omega_{z} \iota(t) v, z\right)^{\beta} \\
& =\tau\left(\begin{array}{cc}
\mathbf{l}_{n} & 0 \\
0 & v(\alpha) 1_{s}
\end{array}\right)\left(f^{y}\right)_{*}\left(\Omega_{z} \cdot{ }^{t} \alpha \iota(t) v, z\right),
\end{aligned}
$$

where $\alpha$ is the projection of $\beta$ to $G_{\mathbf{Q}_{+}}$. This proves (3.38) and completes the proof, since it is obvious that $f^{y}$ is uniquely determined by (3.38).

Proposition 3.11. The action of the elements of $\left(\mathbb{S}\right.$ on $T_{\tau, k, \xi}\left(\mathbf{Q}_{\mathrm{ab}}\right)$ has the following properties:

(i) $(a f+b g)^{y}=a^{y} f^{y}+b^{y} g^{y}$ for $a, b \in \mathbf{Q}_{\mathrm{ab}}$ and $f, g \in T_{\tau, k, \xi}\left(\mathbf{Q}_{\mathrm{ab}}\right)$;

(ii) $\left(f^{x}\right)^{y}=f^{x y}$ for $x, y \in \mathbb{S}$;

(iii) $f^{\beta}=\left.f\right|_{\tau, k, \xi} \beta$ if $\beta \in \mathscr{G}_{\mathbf{Q}}$;

(iv) $f^{\mathrm{u}_{1}(r)}=f^{r}$ if $r \in \mathbf{Z}_{\mathrm{f}}^{\times}$;

(v) $f^{y}=f$ if $f \in T_{\tau, k, \xi}\left(\Delta_{N}, \Lambda ; \mathbf{Q}(e(\mathbf{l} / N))\right)$ and $y \in U\left(S_{N},\{\theta\}\right)$.

Proof. The first four properties follow immediately from the above proof and (3.38). The last one can be proved in exactly the same fashion as Prop. 2.3, since $\left\{y \in \mathfrak{G} \mid f^{y}=f\right\}$ is an open subset of $(S)$, as our definition of $f^{y}$ in the above proof shows.

Notice that Theorem 3.10, together with Proposition 3.11, gives an analogue of the main theorem of [7], which concerns the action of the idele group of an algebraic number field on the theta functions with complex multiplication. Let us now state a consequence of Theorem 3.10 as a theorem in which an abelian variety and its division points are more conspicuous.

Let $\delta$ be a diagonal matrix whose diagonal elements are positive integers $\delta_{1}, \ldots, \delta_{n}$ such that $3 \leqslant \delta_{1}, \delta_{\nu} \mid \delta_{\nu+1}$, and let $L(z, \delta)=z \mathbf{Z}^{n}+\delta \mathbf{Z}^{n}$ for each $z \in \mathfrak{S}_{n}$. We define a projective embedding $\Theta_{z}$ of $\mathbf{C}^{n} / L(z, \delta)$ by

$$
u \mapsto \Theta_{z}(u)=(\theta(u, z ; j, 0))_{j \in \mathfrak{I}} \quad\left(u \in \mathbf{C}^{n}\right)
$$


where $\mathfrak{S}$ is a complete set of representatives for $\delta^{-\mathbf{1}} \mathbf{Z}^{n} / \mathbf{Z}^{n}$. We denote by $A(z, \delta)$ the image variety. Put, for simplicity, $\mathfrak{R}=\mathcal{A}_{0}\left(\mathbf{Q}_{\mathrm{ab}}\right)$. Fix any point $z$ of $\mathfrak{H}_{n}$, and let $B_{\mathfrak{z}}$ be the ring consisting of all elements of $\mathfrak{R}$ holomorphic at $z$, and $\mathfrak{R}_{\mathfrak{z}}$ the field generated over $\mathbf{Q}$ by the values $F(z)$ for all $F \in B_{z}$. The set of all $F$ in $B_{z}$ such that $F(z)=0$ form a unique maximal ideal $P_{z}$ of $B_{z}$, and $F \mapsto F(z)$ gives an isomorphism of $B_{z} / P_{z}$ onto $\Re_{z}$. Now let $x$ be an element of $G_{\mathbf{A}+}$ such that

$$
\left(B_{3}\right)^{x}=B_{z}
$$

Obviously $\left(P_{z}\right)^{x}=P_{z}$, so that $F(z) \mapsto F^{x}(z)$ (with $F \in B_{z}$ ) gives an automorphism of $\Re_{z}$. For example, if $z$ is "generic" for the functions of $\Re,(3.40)$ is satisfied for all $x \in G_{\mathrm{A}_{+}}$. Another extreme case is the points $z$ with complex multiplication, which we shall discuss afterwards. Now we have the following theorem which generalizes [8, Th. 2.4].

THEOREM 3.12. Given an element $x$ of $G_{\mathbf{A}+}$ and a point $z$ of $\mathfrak{S}_{n}$ satisfying (3.40), let $\sigma$ denote the automorphism of $\Re_{3}$ obtained from $x$ as above, and let $R_{\delta}$ be the subgroup of $G_{\mathbf{A}}$ defined by $[8,(1.11)]$. Put $x=q \alpha$ with $q \in R_{\delta}$ and $\alpha \in G_{\mathbf{O}_{+}}$as guaranteed by Lemma 1.1. Put also $\alpha=\left(\begin{array}{ll}a & b \\ c & d\end{array}\right)$ and $\lambda={ }^{t}(c z+d)^{-1}$ with any such $\alpha$ and $q$. Then

(i) $A(\mathfrak{z}, \delta)^{\sigma}=A(\alpha(z), \delta)$,

(ii) $\Theta_{z}\left(\Omega_{z} v\right)^{\sigma}=\Theta_{\alpha(3)}\left(\lambda \Omega_{z} \cdot{ }^{t} x v\right)=\Theta_{\alpha(\xi)}\left(\Omega_{\alpha(3)} \cdot{ }^{t} q v\right)$ for all $v \in\left(\mathbf{A}_{\mathbf{8}}\right)^{2 n}$.

Proof. Put $f_{j}(u, z)=\theta(u, z ; j, 0)$ for each $j \in \mathfrak{F}$. As noted above, $f_{j} \in T_{1 / 2,1}\left(\mathbf{Q}_{\mathrm{ab}}\right)$. Let $y$ and $r$ be elements of $(A)$ lying above $x$ and $q$, respectively. By Th. 3.10, we have

$$
\left(f_{j}\right)_{*}\left(\Omega_{z} v, z\right)^{y}=\left(f_{j}^{y}\right)_{*}\left(\Omega_{z} \cdot{ }^{t} x v, z\right) \quad\left(v \in\left(\mathbf{A}_{\mathbf{i}}\right)^{2 n}, j \in \mathfrak{S}\right)
$$

Put $\Gamma_{\delta}=R_{\delta} \cap G_{\mathbf{Q}}$. This is the group defined in [8, (1.9)]. Let $r=r^{\prime} \iota_{1}(t) \beta$ with $r^{\prime} \in U\left(S_{N},\{\theta\}\right)$, $t \in \mathbf{Z}_{\mathrm{P}}^{\times}$, and $\beta \in \mathscr{B S}_{\mathbf{Q}}$ as in the proof of Theorem 3.10 with a suitable $N$. Then $f_{j}^{r}=f_{j}^{\beta}$, and $\boldsymbol{\beta}$ has an element $\gamma$ of $\Gamma_{\delta}$ as its projection to $G_{\mathbf{Q}_{+}}$. Therefore, by (a.2) of the Appendix, we have $f_{h}^{r} / f_{j}^{r}=f_{h}^{\beta} \mid f_{j}^{\beta}=\left(f_{h} / f_{j}\right)^{\gamma}=f_{h} / f_{j}$ for $h, j \in \mathfrak{F}$. Consequently $\left(f_{h}^{y}\right)_{*} /\left(f_{j}^{y}\right)_{*}=f_{h}^{y} / f_{j}^{y}=\left(f_{h}^{r} / f_{j}^{r}\right) \circ \alpha=$ $\left(f_{h} \circ \alpha\right) /\left(f_{j} \circ \alpha\right)$. Therefore the values of $(3.41)$ are proportional to

$$
f_{j}\left(\alpha\left(\Omega_{z} \cdot{ }^{t} x v, z\right)\right)=f_{j}\left(\lambda \Omega_{z} \cdot{ }^{t} x v, \alpha(z)\right)=f_{j}\left(\Omega_{\alpha(z)} \cdot{ }^{t} q v, \alpha(z)\right)
$$

This proves (ii). Since the points $\Theta_{z}\left(\Omega_{z} v\right)$ with $v \in\left(\mathbf{A}_{\mathbf{f}}\right)^{2 n}$ are dense in $A(z, \delta)$, we obtain (i) from (ii). 
Equality (ii) can also be expressed by the commutative diagram

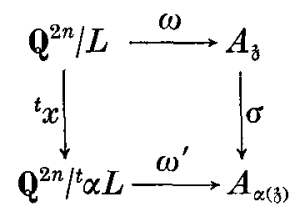

where $L=\left(\begin{array}{ll}1 & 0 \\ 0 & \delta\end{array}\right) \mathbf{Z}^{2 n}, \omega(v)=\Theta_{z}\left(\Omega_{z} v\right)$, and $\omega^{\prime}(v)=\Theta_{\alpha(3)}\left(\Omega_{\alpha(\xi)}{ }^{t}{ }^{t} \alpha^{-1} v\right)$. Notice that ${ }^{t} x L={ }^{t} \alpha L ; \omega$ and $\omega^{\prime}$ are bijective maps onto the groups of all points of finite order on $A_{z}$ and $A_{\alpha(3)}$, respectively.

We insert here a proposition concerning a non-vanishing of an element of $\mathcal{A}_{k}\left(\mathbf{Q}_{\mathrm{ab}}\right)$ at a point $z$ of the above type, which generalizes [8, Lemma 2.5] and is similar to [5, Prop. $10]$.

Proposition 3.13. Suppose that a point $z$ of $\mathfrak{H}_{n}$ and an element $x$ of $G_{\mathrm{A}+}$ satisfy (3.40). If $f \in \mathcal{A}_{k}\left(\mathbf{Q}_{\mathrm{ab}}\right), k \in \mathbf{Z}, f$ is finite at $z$, and $f(z) \neq 0$, then $f^{x}$ is finite at $z$ and $f^{x}(z) \neq 0$.

Proof. This is obvious if $k=0$. The case of negative $k$ can be reduced to positive $k$ by taking $f^{-1}$. Assuming $k>0$, take any non-zero element $g$ of $m_{k}\left(\mathbf{Q}_{\mathrm{ab}}\right)$. Since $G_{\mathbf{Q}_{+}}$is dense in $G_{\mathbf{R}+}$, we can find an element $\alpha$ of $G_{\mathbf{Q}+}$ so that $g(\alpha(z)) \neq 0$. Put $h=g^{\alpha x^{-1}}$. Then $h^{x}(z) \neq 0$, $h / f \in \mathcal{A}_{0}\left(\mathbf{Q}_{\mathrm{ab}}\right)$, and $h / f \in B_{\mathrm{z}}$. By (3.40), $h^{x} / f^{x}=(h / f)^{x} \in B_{\mathfrak{z}}$, and hence $f^{x}(z) \neq 0$. Applying this result to $h^{x}$ and $x^{-1}$, we see that $h(z) \neq 0$. Thus $f / h \in B_{z}$, so that $f^{x} / h^{x} \in B_{z}$, which proves that $f^{x}$ is finite at $z$ and $f^{x}(z) \neq 0$.

Let us now briefly show that the main theorem of [7] can actually be derived from Th. 3.10. Let $z$ be a point of $\mathfrak{S}_{n}$ such that $A(z, \delta)$ has many complex multiplications in the sense of $[7, \S 2]$. Define $Y$ and $K^{\prime}$ as in [7, p. 684], and let $\Phi: Y \rightarrow \mathbf{C}_{n}^{n}$ be the antirepresentation which gives the action of $Y$ on $A(z, \delta)$ as the endomorphism algebra. Define an injection $\varepsilon$ of $Y$ into $\mathbf{Q}_{2 n}^{2 n}$ by $\Phi(a) \Omega_{z}=\Omega_{\vec{b}} \cdot t \varepsilon(\alpha)$ for $a \in Y$, and a map $\eta: K_{\mathbf{A}}^{\prime \times} \rightarrow Y_{\mathbf{A}}^{\times}$by [7, (25)]. Then $\varepsilon \circ \eta$ maps $K_{\mathbf{A}}^{\prime \times}$ into $G_{\mathbf{A}+}$. Given any $r \in K_{\mathbf{A}}^{\prime \times}$, put $x=\varepsilon(\eta(r))^{-1}$. By [4, I, (2.7.3); II, (6.2.3)], we have $\left(B_{3}\right)^{x}=B_{z}$, and

$$
F(z) \in K_{\mathrm{ab}}^{\prime} \quad \text { and } F(z)^{r}=F^{x}(z) \quad \text { for all } F \in B_{\mathrm{z}}
$$

Now let $f \in T_{k, \xi}\left(\mathbf{Q}_{\mathrm{ab}}\right)$. Choose any $h$ of $m_{k}\left(\mathbf{Q}_{\mathrm{ab}}\right)$ so that $h(\mathfrak{z}) \neq 0$, and put $g(u)=f_{*}(u, \mathfrak{z}) / h(\xi)$ for $u \in \mathbf{C}_{s}^{n}$. Then

$$
g\left(\Omega_{\mathrm{z}} v\right)=f_{*}\left(\Omega_{\mathrm{z}} v, z\right) / h(z) \in K_{\mathrm{ab}}^{\prime}
$$


for all $v \in \mathbf{Q}_{s}^{2 n}$ by (3.43), since $f_{*}\left(\Omega_{z} v, z\right) \in m_{k}\left(\mathbf{Q}_{\mathrm{ab}}\right)$. Take an element $y$ of (S) lying above $x$, and put $g^{\prime}(u)=\left(f^{y}\right)_{*}(u, z) / h^{y}(z)$. This is meaningful since $h^{y}(z) \neq 0$ by Prop. 3.13. By (3.43) and Th. 3.10, we have

and thus

$$
\begin{aligned}
g\left(\Omega_{\mathrm{z}} v\right)^{r} & =\left.\left[f_{*}\left(\Omega_{z} v, z\right) / h(z)\right]^{x}\right|_{z=\mathfrak{z}}=\left(f^{y}\right)_{*}\left(\Omega_{\mathfrak{z}} \cdot{ }^{t} x v, \mathfrak{z}\right) / h^{y}(\mathfrak{z}) \\
& =g^{\prime}\left(\Omega_{\mathfrak{z}} \cdot{ }^{t} x v\right)=g^{\prime}\left(\Phi\left(\eta(r)^{-1}\right) \Omega_{\mathfrak{z}} v\right)
\end{aligned}
$$

$$
g(u)^{r}=g^{\prime}\left(\Phi\left(\eta(r)^{-1}\right) u\right) \quad \text { for all } u \in \Omega_{\mathfrak{z}} Q_{s}^{2 n} \quad \text { and all } r \in K_{\mathbf{A}}^{\prime \times} .
$$

When $s=1$ and $k=1 / 2$, this is exactly the fundamental relation needed for the proof of the main theorem of [7]. Since the functions $h(z)^{-1} f^{*}$ span the space of "arithmetic theta functions" as shown by [7, Prop. 2.5], we obtain the theorem.

\section{Proof of Proposition 3.8}

Given a lattice $Y$ and an element $\zeta$ in the vector space $\left\{\left.\eta \in \mathbf{Q}_{n}^{n}\right|^{t} \eta=\eta\right\}$ and a lattice $L$ in $\mathbf{Q}_{n}^{s}$, consider all formal series of the form

$$
p(u, z)=\sum_{\zeta \leqslant \eta \in Y} \sum_{\lambda \in L} a(\eta, \lambda) e_{n}(\eta z+u \lambda) \quad\left(u \in \mathbb{C}_{s}^{n}, z \in \mathfrak{S}_{n}\right)
$$

with complex coefficients $a(\eta, \lambda)$ satisfying the following condition:

$$
\text { For a fixed } \eta \text {, there are only finitely many } \lambda \text { such that } a(\eta, \lambda) \neq 0 \text {. }
$$

As proved in Prop. 3.1, the components of an element of $T_{r, k, \xi}$ satisfy (4.2). Let $\Phi$ denote the set of all such formal series, with all choices of $Y$ and $L$. Obviously $\Phi$ forms a ring. Take linearly independent positive definite symmetric elements $\zeta_{1}, \ldots, \zeta_{M}$ of $\mathbf{Q}_{n}^{n}$, where $M=n(n+1) / 2$. To each $(\eta, \lambda)$ we can assign its "coordinates"

$$
\left(\operatorname{tr}\left(\zeta_{1} \eta\right), \ldots, \operatorname{tr}\left(\zeta_{M} \eta\right), \lambda_{11}, \lambda_{12}, \ldots, \lambda_{s n}\right)
$$

By means of these coordinates, we introduce a lexicographic order into the set of all $(\eta, \lambda)$. Then, for $0 \neq p \in \Phi$, the "first" non-vanishing coefficient of $p$ is meaningful. Therefore $\Phi$ is an integral domain.

LEMMA 4.1. Suppose $p, q, r \in \Phi, p q=r, p \neq 0, p$ has coefficients in a subfield $F$ of $\mathbf{C}$, and $r$ has coefficients in a vector space $W$ over $F$. Then $q$ has coefficients in $W$.

This can be easily proved by means of the above lexicographic order.

Our proof of Prop. 3.8 needs special care when $n=1$, since the holomorphy on $\mathfrak{H}_{1}$ does not guarantee the finiteness at cusps. To avoid the difficulty, let $S_{k, \xi}(\Delta, \Lambda)$ denote the 
set of all holomorphic $f$ satisfying (3.10) and (3.11) with trivial $\tau$ such that $f_{*}\left(\Omega_{z} v, z\right)$ belongs, as a function of $z$, to $\mathcal{A}_{k}(\mathbb{C})$ for all $v \in Q_{s}^{2 n}$. Then we define $\mathcal{S}_{k, \xi}(\Re)$ for a subfield $\Re$ of $\mathbf{C}$ to be the set of all such $f$, with any possible $\Delta$ and $\Lambda$, and with Fourier coefficients (i.e., $c(\eta, \lambda)$ of $(3.12)$ ) in $\Re$. Obviously $S=T$ if $n>1$.

LEMMA 4.2. Let $\mathcal{C}_{k}(\Re)$ denote the set of all elements of $\mathcal{A}_{k}(\Re)$ holomorphic on $\mathfrak{H}_{1}$ (but not necessarily so at cusps). Then $\mathcal{L}_{k}(\mathbf{C})=\mathcal{L}_{k}(\mathbf{Q}) \otimes_{\mathbf{Q}} \mathbf{C}$.

Proof. Given $f \in \mathcal{L}_{k}(\mathbf{C})$, consider a cusp form

$$
\eta(z)=e(z / 24) \prod_{n=1}^{\infty}(1-e(n z))
$$

and observe that $\eta^{2 h} f \in m_{k+n}(\mathbf{C})$ for a suitable positive integer $h$. Therefore $\eta^{2 h} f$ is a finite C-linear combination of elements of $m_{k+h}(\mathbf{Q})$, Dividing this by $\eta^{2 h}$, we obtain our lemma.

Lемм А 4.3. If $n=1$ and $f \in S_{k, \xi}(\mathbf{C})$, then

$$
f(u, z)=\sum_{j=1}^{N} c_{j}(z) F_{j}(u, z)
$$

with $c_{i} \in \mathcal{L}_{k-(r / 2)}(\mathbf{C})$ and $F_{j} \in T_{r / 2, \xi}(\mathbf{Q})$, where $r=\operatorname{rank}(\xi)$, and hence $f$ has a Fourier expansion of type (4.1).

Proof. The proof of Prop. 3.5 shows at least an expression (4.3) for $f$ with $c_{j}$ holomorphic on $\mathfrak{S}_{1}$ and functions $F_{j} \in T_{r / 2, \xi}(\mathbf{Q})$ such that $F_{j}\left(u, z_{0}\right)$ for $j=1, \ldots, N$ are linearly independent over $\mathbf{C}$ for every $z_{0}$. Put, for each $v \in \mathbf{Q}_{s}^{2}$ and for any fixed $z_{0}$,

$$
X(v)=\left\{\left(b_{1}, \ldots, b_{N}\right) \in \mathbf{C}_{N}^{1} \mid \sum_{j=1}^{N} b_{j} F_{j}\left(\Omega_{z_{\mathrm{o}}}, z_{0}\right)=0\right\} .
$$

The intersection of all such $X(v)$ is $\{0\}$, and hence we can find $N$ elements $v_{1}, \ldots, v_{N}$ of $\mathbf{Q}_{s}^{2}$ such that $\bigcap_{i=1}^{N} X\left(v_{i}\right)=\{0\}$. This implies that

$$
\operatorname{det}\left(\left(F_{j}\right)_{*}\left(\Omega_{z} v_{i}, z\right)\right)_{i, j} \neq 0 .
$$

Denote this determinant by $D(z)$. The equations

$$
f_{*}\left(\Omega_{z} v_{i}, z\right)=\sum_{j=1}^{N} c_{j}(z)\left(F_{j}\right)_{*}\left(\Omega_{z} v_{i}, z\right) \quad(i=1, \ldots, N)
$$

show that $D c_{j} \in \mathcal{A}_{l}(\mathbf{C})$ with $l=k+(N-1) r / 2$. Since $D \in M_{r N / 2}(\mathbf{C})$, we obtain the desired conclusion. 
Now, to prove Prop. 3.8, we may assume, in view of Prop. 3.3, that $\xi$ is non-degenerate. Obviously any elements of $T_{\tau, k, \xi}(\mathbf{Q})$ linearly independent over $\mathbf{Q}$ are also linearly independent over $\mathbf{C}$. Therefore our aim is to show that $T_{\tau, k, \xi}$ is spanned by $T_{\tau, k, \xi}(\mathbf{Q})$ over C. Let $f \in T_{\tau, k, \xi}$, and let $W_{f}$ be the vector space spanned over $\mathbf{Q}$ by the Fourier coefficients of the components of $f$. Let us first prove

$$
W_{f} \text { is finite-dimensional over } \mathbf{Q} \text {; }
$$

$$
\text { If }\left\{r_{\nu}\right\} \text { is a basis of } W_{f} \text { over } \mathbf{Q} \text { and } \tau \text { is trivial, then } f=\sum_{\nu} r_{\nu} g_{\nu} \text { with } g_{\nu} \in S_{k, \xi}(\mathbf{Q}) \text {. }
$$

If $\tau$ is trivial, we see, from Prop. 3.5 and Prop. 2.1 when $n>1$ and from Lemmas 4.2 and 4.3 when $n=1$, that $f$ is a finite sum $\sum t_{x} f_{x}$ with $t_{x} \in \mathbf{C}$ and $f_{x} \in S_{k, \xi}(\mathbf{Q})$. Therefore $W_{f}$ is contained in $\sum \mathbf{Q} t_{x}$ and hence finite-dimensional. Given a basis $\left\{r_{\nu}\right\}$ of $W_{f}$, take a basis of $\sum \mathbf{Q} t_{\varkappa}$ of the form $\left\{r_{\nu}\right\} \cup\left\{r_{\mu}^{\prime}\right\}$ and express each $t_{\kappa}$ as a $\mathbf{Q}$-linear combination of $r_{\nu}$ and $r_{\mu}^{\prime}$. Then $f=\sum r_{\nu} g_{\nu}+\sum r_{\mu}^{\prime} g_{\mu}^{\prime}$ with $g_{\nu}$ and $g_{\mu}^{\prime}$ in $S_{k, \xi}(\mathbf{Q})$. Since $f$ has coefficients in $W_{f}$, comparison of coefficients shows that $g_{\mu}^{\prime}$ must be 0 , and thus $f=\sum r_{\nu} g_{\nu}$, which proves (4.5).

In the general case with an arbitrary $\tau$, take $A$ as in Prop. 3.7 with any point $\left(u_{0}, z_{0}\right)$, and put $d=\operatorname{det}(A), B=d \cdot{ }^{t} A^{-1}$, and $g={ }^{t} B f$ for a given $f \in T_{\tau, k, \xi}$. Then the components of $g$ belong to $T_{l, m \xi}$ with some $l$, and $W_{g} \subset W_{f}$. By Lemma 4.1, the relation $d f=A g$ shows that $W_{f} \subset W_{g}$, so that $W_{f}=W_{g}$. Since (4.4) is true for trivial $\tau$, we have (4.4) for an ar-

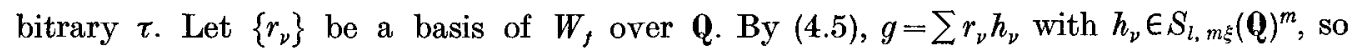
that $f=\sum r_{\nu} d^{-1} A h_{\nu}$. Note that $d\left(u_{0}, z_{0}\right) \neq 0$. Given another point $\left(u_{1}, z_{1}\right)$, we take $A_{1}, B_{1}$, and $d_{1}$ similarly so that $d_{1}\left(u_{1}, z_{1}\right) \neq 0$, and obtain an expression $f=\sum r_{\nu} d_{1}^{-1} A_{1} h_{1 v}$ with $h_{1 \nu} \in S_{l, m \xi}(\mathbf{Q})^{m}$ and with the same $r_{\nu}$. Obviously

$$
\sum_{\nu} r_{\nu}\left(d A_{1} h_{1 \nu}-d_{1} A h_{\nu}\right)=0
$$

Now $d A_{1} h_{1 \nu}$ and $d_{1} A h_{\nu}$ have Fourier expansions of type (4.1). Since the components of $A, A_{1}, d$, and $d_{1}$ satisfy (4.2), each sum expressing a Fourier coefficient of $d A_{1} h_{1 \nu}$ or $d_{1} A h_{\nu}$ in terms of those of $d, d_{1}, A, A_{1}, h_{\nu}$, and $h_{1 \nu}$ is a finite sum, so that $d A_{1} h_{1 \nu}$ and $d_{1} A h_{\nu}$ have coefficients in $\mathbf{Q}^{m}$. Since $\left\{r_{\nu}\right\}$ are linearly independent over $\mathbf{Q}$, we have $d A_{1} h_{1 \nu}=d_{1} A h_{\nu}$, and so $d^{-1} A h_{\nu}=d_{1}^{-1} A_{1} h_{1 \nu}$. This shows that $d^{-1} A h_{\nu}$ is holomorphic on the whole $\mathbf{C}_{s}^{n} \times \mathfrak{F}_{n}$. Obviously it satisfies (3.10) and (3.11). For every automorphism $\sigma$ of $\mathbf{C}$, define a holomorphic function $f_{\sigma}$ by $f_{\sigma}=\sum_{\nu} r_{\nu}^{\sigma} d^{-1} A h_{\nu}$. Both $d f$ and $A h_{\nu}$ have Fourier expansions of type (3.12). Define the action of $\sigma$ on any such series by (3.37), which, in general, is only a formal series. Since $A h_{\nu}$ has coefficients in $\mathbf{Q}^{m}$ for the same reason as above, we have $(d f)^{\sigma}=\sum r_{\nu}^{\sigma} A h_{\nu}=d f_{\sigma}$, and thus $(d f)^{\sigma}$ is meaningful as a function. Let $t$ be an element of 
$\mathbf{Z}_{\mathrm{f}}^{\times}$whose action on $\mathbf{Q}_{\mathrm{ab}}$ coincides with $\sigma$. Then the proof of Prop. 3.9 is applicable to both $d$ and $d f$, so that for $v \in \mathbf{Q}_{s}^{2 n}$, we have

$$
\begin{gathered}
d_{*}\left(\Omega_{z} \iota(t) v, z\right)=d_{*}\left(\Omega_{z} v, z\right)^{\sigma} \\
\left(d f_{\sigma}\right)_{*}\left(\Omega_{z} \iota(t) v, z\right)=\left((d f)^{\sigma}\right)_{*}\left(\Omega_{z} \iota(t) v, z\right)=(d f)_{*}\left(\Omega_{z} v, z\right)^{\sigma} .
\end{gathered}
$$

Since $(d f)_{*}=d_{*} f_{*}$ and $\left(d f_{\sigma}\right)_{*}=d_{*}\left(f_{\sigma}\right)_{*}$, we have

$$
\left(f_{\sigma}\right)_{*}\left(\Omega_{z} \iota(t) v, z\right)=f_{*}\left(\Omega_{z} v, z\right)^{\sigma}
$$

if $d_{*}\left(\Omega_{z} v, z\right) \neq 0$. For every $v \in \mathbf{Q}_{s}^{2 n}$, we can take $A$ so that $d_{*}\left(\Omega_{z} v, z\right) \neq 0$, and so we have (4.6) for all $v \in \mathbf{Q}_{s}^{2 n}$. (Note that $f_{\sigma}$ is independent of the choice of $A$.) This shows that $f_{\sigma}$ satisfies (3.23), i.e., $f_{\sigma} \in T_{\tau, k, \xi}$. Now, by Lemma 4.4 below, we can find a set of automorphisms $\{\sigma\}$ such that $\operatorname{det}\left(r_{\nu}^{\sigma}\right)_{\sigma, \nu} \neq 0$. Then the relations $f_{\sigma}=\sum r_{\nu}^{\sigma} d^{-1} A h_{\nu}$ show that $d^{-1} A h_{\nu} \in$ $T_{\tau, k, \xi}$. By Lemma 4.1, we obtain $d^{-1} A h_{\nu} \in T_{\tau, k, \xi}(\mathbf{Q})$, which completes the proof.

LEMMA 4.4. If $r_{1}, \ldots, r_{N}$ are $N$ complex numbers linearly independent over $\mathbf{Q}$, then there is a set $\{\sigma\}$ of $N$ automorphisms of $\mathbf{C}$ such that $\operatorname{det}\left(r_{\nu}^{\sigma}\right)_{\sigma, \nu} \neq 0$.

Proof. Let $H$ denote the linear subspace of $\mathbf{C}_{N}^{1}$ consisting of all $\left(x_{1}, \ldots, x_{N}\right)$ of $\mathbf{C}_{N}^{1}$ such that $\sum_{v=1}^{N} r_{v}^{\sigma} x_{\nu}=0$ for all automorphisms $\sigma$ of $\mathbf{C}$. Obviously $H$ is stable under the map $\left(x_{1}, \ldots, x_{N}\right) \mapsto\left(x_{1}^{\sigma}, \ldots, x_{N}^{\sigma}\right)$ for all $\sigma$. Therefore $H$ is defined over $\mathbf{Q}$. If $H \neq\{0\}, H$ contains a non-zero vector of $\mathbf{Q}_{N}^{\mathbf{1}}$, which contradicts the linear independence of $\left\{r_{\nu}\right\}$. Hence $H=\{0\}$, from which our assertion follows.

\section{Proof of Theorem 1.2}

We shall now complete the proof of Theorem 1.2 in the case of non-trivial $\varrho$. Observe that the theory of $\S 3$ concerning $T_{k, \xi}$ is independent of Theorem 1.2 with non-trivial $\varrho$. Consider $T_{k, \xi}$ with $s=1$ and $0<\xi \in \mathbf{Q}$. Given an element $f(u, z)$ of $T_{k, \xi}$, define a $\mathbf{C}^{n}$-valued function $D f$ on $\mathfrak{S}_{n}$ by

$$
(D f)(z)=\frac{1}{2 \pi i}\left(\begin{array}{c}
\left(\partial f / \partial u_{1}\right)(0, z) \\
\vdots \\
\left(\partial f / \partial u_{n}\right)(0, z)
\end{array}\right) \quad\left(z \in \mathfrak{S}_{n}\right)
$$

We see easily that, for every $\beta=(\alpha, \psi) \in \mathscr{S}_{\mathbf{Q}}$ with $\alpha=\left(\begin{array}{ll}a & b \\ c & d\end{array}\right)$,

$$
\left.(D f)\right|_{k} \beta=(c z+d) D\left(f^{\beta}\right),
$$

and hence $D f \in m_{\ell, k}(\mathbf{C})$, where $\varrho(X)=X$. If $\sigma$ is an automorphism of $\mathbf{C}$, we have obviously

$$
(D f)^{\sigma}=D\left(f^{\sigma}\right) \text {. }
$$


Given $n$ elements $f_{1}, \ldots, f_{n}$ of $T_{k, \xi}$, we consider a $\mathbf{C}_{n^{-}}^{1}$ valued function $\mathfrak{f}=\left(f_{1}, \ldots, f_{n}\right)$ on $\mathbf{C}_{1}^{n} \times \mathfrak{H}_{n}$, and put

$$
D \mathfrak{f}=\left(D f_{1}, \ldots, D f_{n}\right)
$$

Formulas (5.2) and (5.3) hold also with $\mathfrak{f}$ in place of $f$. We call $\mathfrak{f}$ regular if $\operatorname{det}(D \mathfrak{f})$ is not identically equal to 0 .

Proposition 5.1. (i) For every $z_{0} \in \mathfrak{S}_{n}$, there exists a regular $\mathfrak{f}=\left(f_{1}, \ldots, f_{n}\right)$ with $f_{\nu}$ in $T_{1 / 2,1}(\mathbf{Q})$ such that $\operatorname{det}(D \tilde{\mathfrak{f}})\left(z_{0}\right) \neq 0$.

(ii) Suppose that $f_{\nu} \in T_{k, \xi}(\mathbf{Q})$ and $\mathfrak{f}=\left(f_{1}, \ldots, f_{n}\right)$ is regular. Then $\mathfrak{f}^{y}=\left(f_{1}^{y}, \ldots, f_{n}^{y}\right)$ is regular for every $y \in(\mathfrak{s}$.

(iii) If $\mathfrak{f}$ is as in (ii) and $\mathfrak{h}=\left(h_{1}, \ldots, h_{n}\right)$ with $h_{\nu} \in T_{k, \xi}\left(\mathbf{Q}_{\mathrm{ab}}\right)$, then $D\left(\mathfrak{f}^{y}\right)^{-1} D\left(\mathfrak{h}^{y}\right)=\left((D \mathfrak{f})^{-1} D \mathfrak{h}\right)^{y}$ for every $y \in \mathfrak{B S}$.

(In the last relation, $(D \mathfrak{F})^{-1} D \mathfrak{h}$ has components in $\mathcal{A}_{0}\left(\mathbf{Q}_{\mathrm{ab}}\right)$, so that $\left((D \mathfrak{f})^{-1} D \mathfrak{h}\right)^{y}$ is meaning$f u l$.

Proof. Assertion (i) follows immediately from [8, Prop. 1.2] and its proof. Assertion (ii) is obvious, since $\mathfrak{f}^{y}=\mathfrak{f}^{\beta}$ for some $\beta \in \mathfrak{G S}_{\mathbf{Q}}$, as shown in the proof of Th. 3.10. To prove (iii), put $E=(D \mathfrak{f})^{-1} D \mathfrak{h}$. We can find $\sigma \in \mathrm{Gal}\left(\mathbf{Q}_{\mathrm{ab}} / \mathbf{Q}\right)$ and $\beta \in \mathfrak{S S}_{\mathbf{Q}}$ such that $\left.\mathfrak{f}^{y}=\mathfrak{f}^{\beta}, \mathfrak{h}^{y}=(\mathfrak{h})^{\sigma}\right)^{\beta}$, and $E^{y}=\left(E^{\sigma}\right)^{\beta}$. Then

$$
\begin{aligned}
\left((D \mathfrak{f})^{-1} D \mathfrak{h}\right)^{y} & =\left(\left((D \mathfrak{f})^{-1} D \mathfrak{h}\right)^{\sigma}\right)^{\beta}=\left(\left((D \mathfrak{f})^{\sigma}\right)^{\beta}\right)^{-1}\left((D \mathfrak{h})^{\sigma}\right)^{\beta} \\
& \left.=D\left(\left(\mathfrak{f}^{\sigma}\right)^{\beta}\right)^{-1} D\left((\mathfrak{h})^{\sigma}\right)^{\beta}\right)=D\left(\mathfrak{f}^{y}\right)^{-1} D\left(\mathfrak{h}^{y}\right)
\end{aligned}
$$

by (5.2) and (5.3). Q.E.D.

Proposition 5.2. For every rational representation $\varrho$ of $G L_{n}(\mathbf{Q})$ and $k \in 2^{-1} \mathbf{Z}$, we have $m_{\varrho, k}(\mathbf{C})=m_{\varrho, k}(\mathbf{Q}) \otimes \mathbf{Q} \mathbf{C}$.

Proof. Let $m$ be the degree of $\varrho$. For every $z_{0} \in \mathfrak{S}_{n}$, there exist a positive integer $j$ and a $\mathbf{C}_{m}^{m}$-valued function $A$ on $\mathscr{S}_{n}$ such that:

(i) the columns of $A$ belong to $m_{Q, j}(\mathbf{Q})$;

(ii) $\operatorname{det}\left(A\left(z_{0}\right)\right) \neq 0$.

This follows either from Prop. 3.7 or from (i) of Prop. 5.1. With any such $A$ and given $f \in m_{\varrho, k}(\mathbf{C})$, put $d=\operatorname{det}(A), B=d \cdot{ }^{t} A^{-1}$, and $g={ }^{t} B f$. Then the components of $g$ belong to $m_{t}(\mathbf{C})$ for some $t$. Since $m_{t}(\mathbf{C})=m_{t}(\mathbf{Q}) \otimes_{\mathbf{Q}} \mathbf{C}$ by Prop. 2.1, we obtain our assertion by the same type of reasoning as in the proof of Prop. 3.8. 
This proposition shows that the action of an automorphism $\sigma$ of $\mathbf{C}$ on $m_{Q, k}(\mathbf{C})$ defined by (1.4) actually maps $m_{Q, k}(\mathbf{C})$ onto itself.

To define the action of $G_{\mathrm{A}+}$ on $\mathcal{A}_{\varrho}\left(\mathbf{Q}_{\mathrm{ab}}\right)$, first assume $\varrho(X)=X$. Choose any regular $\mathfrak{F}=\left(f_{1}, \ldots, f_{n}\right)$ with $f_{\nu}$ in $T_{1 / 2,1}(\mathbf{Q})$. Given $g \in \mathcal{A}_{\varrho}\left(\mathbf{Q}_{\mathrm{ab}}\right)$, observe that the components of $(D \mathfrak{f})^{-1} g$ belong to $\mathcal{A}_{1 / 2}\left(\mathbf{Q}_{\mathrm{ab}}\right)$, so that $\left((D \mathfrak{F})^{-1} g\right)^{y}$ is meaningful for $y \in(3)$. Now for $x \in G_{\mathrm{A}_{+}}$, define $g^{x}$ by $g^{x}=D\left(\mathfrak{f}^{y}\right)\left((D \tilde{f})^{-1} g\right)^{y}$ with any element $y$ of $\left(\mathfrak{S}\right.$ with projection $x$ on $G_{\mathbf{A}+}$ of the form $y=(x, 1, v)$. This is independent of the choice of $y$, and also of the choice of $f$ because of (iii) of Prop. 5.1. If $\varrho(X)=X \otimes \ldots \otimes X$, then considering $D \mathfrak{f} \otimes \ldots \otimes D \mathfrak{f}$ instead of $D \mathfrak{f}$, we can similarly define the action of $G_{\mathbf{A}+}$ on $\mathcal{A}_{\varrho}\left(\mathbf{Q}_{\mathrm{ab}}\right)$. Since every irreducible rational representation of $G L_{n}(\mathbf{Q})$ is obtained as a suitable power of determinant times a $\mathbf{Q}$-rational constituent of such a tensor representation, it is now easy to define the action of $G_{\mathbf{A}}$ on $\mathcal{A}_{0}\left(\mathbf{Q}_{\mathrm{ab}}\right)$ with an arbitrary $\varrho$. Then the properties (i-viii) of Theorem 1.2 can be verified in a straightforward way. In particular, to prove the associativity (iii), we need (iii) of Prop. 5.1.

\section{Partial Fourier expansions of modular forms}

Let $n$ and $s$ be positive integers. We shall now show that a certain Fourier expansion of a modular form on $\mathfrak{S}_{n+s}$ yields naturally some elements of $T_{\tau, k, \xi}$. First we write the variable point $Z$ of $\mathfrak{S}_{n+s}$ in the form

$$
Z=\left(\begin{array}{cc}
z & u \\
t_{u} & w
\end{array}\right) \quad \text { with } z \in \mathfrak{W}_{n}, w \in \mathfrak{W}_{s}, u \in \mathbf{C}_{s}^{n}
$$

We define the objects $G$, (SS, $\mathcal{A}_{\ell, k}, m_{\varrho, k}, \Gamma_{N}$, etc. with degree $n+s$ instead of $n$, and denote them by $G^{\prime}, G^{\prime}, \mathcal{A}_{Q, k}^{\prime}, m_{Q, k}^{\prime}, \Gamma_{N}^{\prime}$, etc. Let us now consider the elements of $G_{Q}^{\prime}$ of the form

$$
\gamma=\left(\begin{array}{cccc}
1_{n} & 0 & 0 & q \\
{ }^{t} p & \mathbf{1}_{s} & { }^{t} q & r \\
0 & 0 & 1_{n} & -p \\
0 & 0 & 0 & 1_{s}
\end{array}\right), \quad p \in \mathbf{Q}_{s}^{n}, q \in \mathbf{Q}_{s}^{n}, r \in \mathbf{Q}_{s}^{s}
$$

Such a $\gamma$ belongs to $G_{\mathbf{Q}}^{\prime}$ if and only if ${ }^{t} q p+r$ is symmetric. For $Z$ as in (6.1), we have

$$
\gamma(Z)=\left(\begin{array}{cc}
z & u+z p+q \\
{ }^{t} u+{ }^{t} p z+{ }^{t} q & w+{ }^{t} p z p+{ }^{t} p u+{ }^{t} u p+{ }^{t} q p+r
\end{array}\right) .
$$

Let $\tau$ be a polynomial representation $G L_{n+s}(\mathbf{Q}) \rightarrow G L_{m}(\mathbf{Q})$ as in $\S 3$, and let $F(Z)=F(z, u, w)$ be an element of $m_{\tau, k}^{\prime}(\mathbf{C})$ with $0 \leqslant k \in 2^{-1} \mathbf{Z}$. Then we have a Fourier expansion of the form

$$
F(z, u, w)=\sum_{\xi} f_{\xi}(u, z) e_{s}\left(\frac{1}{2} \xi w\right)
$$


with holomorphic $\mathbf{C}^{m}$-valued functions $f_{\xi}(u, z)$ on $\mathbf{C}_{s}^{n} \times \mathfrak{S}_{n}$, where $\xi$ runs over non-negative elements of a lattice in $\left\{\left.\boldsymbol{\xi} \in \mathbf{Q}_{s}^{s}\right|^{t} \xi=\xi\right\}$. Notice that $f_{\xi}$ is defined on the whole $\mathbf{C}_{s}^{n} \times \mathfrak{H}_{n}$, though $F(Z)$ is defined only for $\operatorname{Im}(Z)>0$. Given $\alpha=\left(\begin{array}{ll}a & b \\ c & d\end{array}\right) \in G$, define an element $\alpha^{\prime}$ of $G^{\prime}$ by

$$
\alpha^{\prime}=\left(\begin{array}{cccc}
a & 0 & b & 0 \\
0 & 1_{s} & 0 & 0 \\
c & 0 & d & 0 \\
0 & 0 & 0 & v(\alpha) \mathbf{l}_{s}
\end{array}\right)
$$

The map $\alpha \mapsto \alpha^{\prime}$ is a Q-rational injection, so that it can be extended to a continuous map of $G_{\mathbf{A}}$ into $G_{\mathbf{A}}^{\prime}$. With $\alpha \in G_{\mathbf{Q}+}$ and $\alpha^{\prime}$ as in (6.5), we have

$$
\begin{gathered}
\alpha^{\prime}(Z)=\left(\begin{array}{cc}
\alpha(z) & { }^{t}(c z+d)^{-1} u \\
t u(c z+d)^{-1} & v(\alpha)^{-1} w-v(\alpha)^{-1} \cdot{ }^{t} u(c z+d)^{-1} c u
\end{array}\right), \\
\left(\begin{array}{cc}
c & 0 \\
0 & 0
\end{array}\right) Z+\left(\begin{array}{cc}
d & 0 \\
0 & v(\alpha) 1_{s}
\end{array}\right)=\left(\begin{array}{cc}
c z+d & c u \\
0 & v(\alpha) 1_{s}
\end{array}\right), \\
F\left(\alpha^{\prime}(Z)\right)=\sum_{\xi} f_{\xi}(\alpha(u, z)) e_{s}\left(-\frac{1}{2} v(\alpha)^{-1} \xi \cdot{ }^{t} u(c z+d)^{-1} c u\right) e_{s}\left(\frac{1}{2} v(\alpha)^{-1} \xi w\right) .
\end{gathered}
$$

If $\iota^{\prime}$ is the map of $\mathbf{A}_{+}^{\times}$into $G_{\mathbf{A}^{+}}^{\prime}$ corresponding to $\iota$, we see that

$$
\iota(t)^{\prime}=\iota^{\prime}(t) \quad\left(t \in \mathbf{A}_{+}^{\times}\right) .
$$

Let $(\alpha, \psi) \in \mathfrak{G S}_{\mathbf{Q}}$ with $\alpha=\left(\begin{array}{ll}a & b \\ c & d\end{array}\right)$. Define a holomorphic function $\psi^{\prime}$ on $\mathfrak{S}_{n+s}$ by

$$
\psi^{\prime}\left(\begin{array}{cc}
z & u \\
{ }^{u} u & w
\end{array}\right)=v(\alpha)^{s / 2} \psi(z)
$$

From (6.7), we know that $\left(\alpha^{\prime}, \psi^{\prime}\right) \in \mathfrak{G}_{\mathbf{Q}}^{\prime}$, and thus obtain an injection

$$
(\alpha, \psi) \mapsto\left(\alpha^{\prime}, \psi^{\prime}\right)
$$

of $G_{\mathbf{Q}}$ into $G_{\mathbf{Q}}^{\prime}$.

A good example of Fourier expansion (6.4) can be obtained from theta functions as follows. Let $\theta^{\prime}$ denote the function defined by (3.26) with $n+s$ instead of $n$, and put $\Phi(Z)=$ $\theta^{\prime}(0, Z ; p, q)$ with

$$
p=\left(\begin{array}{l}
p_{1} \\
p_{2}
\end{array}\right), q=\left(\begin{array}{l}
q_{1} \\
q_{2}
\end{array}\right) \quad\left(p_{1}, q_{1} \in \mathbf{Q}^{n} ; p_{2}, q_{2} \in \mathbf{Q}^{s}\right)
$$

Then

$$
\Phi(Z)=\sum_{y-p_{2} \in Z^{*}} e\left({ }^{t} y q_{2}\right) \theta\left(u y, z ; p_{1}, q_{1}\right) e\left(\frac{1}{2} \cdot{ }^{t} y w y\right)
$$


Thus the coefficients $f_{\xi}$ for $\Phi$ are linear combinations of $\theta\left(u y, z ; p_{1}, q_{1}\right)$. In particular, if we put $\theta^{\prime}(Z)=\theta^{\prime}(0, Z ; 0,0)$, then

$$
\theta^{\prime}(Z)=\sum_{y \in Z^{s}} \theta(u y, z ; 0,0) e\left(\frac{1}{2} \cdot{ }^{t} y w y\right)
$$

Define $\Gamma_{\theta}$ and $\lambda(\gamma)$ for $\gamma \in \Gamma_{\theta}$ by (a.1) and (a.2) of the Appendix, and similarly a subgroup $\Gamma_{\theta}^{\prime}$ of $G_{Q}^{\prime}$ and $\lambda^{\prime}(\beta)$ for $\beta \in \Gamma_{\theta}^{\prime}$. From (6.8), (6.12), and (a.2), we see easily that $\lambda(\gamma)=\lambda^{\prime}\left(\gamma^{\prime}\right)$ for every $\gamma \in \Gamma_{\theta}$, or more precisely,

$$
\theta^{\prime}\left(\gamma^{\prime}(Z)\right) / \theta^{\prime}(Z)=\theta(\gamma(z)) / \theta(z) \quad \text { for every } \gamma \in \Gamma_{\theta}
$$

Therefore, if $\Delta_{N}$ and $\Delta_{N}^{\prime}$ are the images of $\Gamma_{N}$ and $\Gamma_{N}^{\prime}$ under the map (2.7) (respectively with $n$ and $n+s)$, then the map (6.11) of $\mathscr{G}_{\mathbf{Q}}$ into $\mathscr{G}_{\mathbf{Q}}^{\prime}$ sends $\Delta_{N}$ into $\Delta_{N}^{\prime}$.

Proposition 6.1. Suppose $F \in \mathcal{M}_{\tau, k}^{\prime}\left(\Gamma_{N}^{\prime}, \mathfrak{R}\right)$ or $F \in \mathcal{M}_{\tau, k}^{\prime}\left(\Delta_{N}^{\prime}, \mathfrak{R}\right)$ according as $2 k$ is even or odd, where $\mathfrak{R}$ is a subfield of $\mathbf{C}$. Then the Fourier coefficient $f_{\xi}$ of $F$ defined by $(6.4)$ belongs to $T_{\tau, k, \xi}\left(\Gamma_{N}, \Lambda ; \Re\right)$ or $T_{\tau, k, \xi}\left(\Delta_{N}, \Lambda ; \Re\right)$ accordingly, with a suitable $\Lambda$.

Proof. Let $F \in \mathcal{M}_{r, k}^{\prime}\left(\Delta_{N}^{\prime}, \mathfrak{R}\right)$. If $\delta=(\gamma, 1) \in \mathbb{G}_{\mathbf{Q}}$ with $\gamma$ defined by $(6.2)$ and ${ }^{t} q p+r=0$, formula (6.3) shows that

$$
F^{\delta}(z, u, w)=\tau\left(\begin{array}{ll}
1_{n} & p \\
0 & 1_{s}
\end{array}\right) \sum_{\xi} e_{s}\left(\xi\left(\frac{1}{2} \cdot{ }^{t} p z p+{ }^{t} p u\right)\right) f_{\xi}(u+z p+q, z) e_{s}\left(\frac{1}{2} \xi w\right)
$$

If $\beta=(\alpha, \psi) \in \mathscr{S}_{\mathbf{Q}}^{\prime}$ and $\beta^{\prime}=\left(\alpha^{\prime}, \psi^{\prime}\right) \in \mathbb{S}_{\mathbf{Q}}^{\prime}$ with $\alpha^{\prime}$ of $(6.5)$ as above, we obtain, from (6.8),

$$
F^{\beta^{\prime}}(z, u, w)=v(\alpha)^{-k s} \sum_{\xi}\left(f_{\xi}\right)^{\beta}(u, z) e_{s}\left(\frac{1}{2} v(\alpha)^{-1} \xi w\right)
$$

Therefore $f_{\xi}$ satisfies (3.10) and (3.11) with a suitable $\Lambda$ and $\Delta=\Delta_{N}$. Obviously $f_{\xi}$ has Fourier coefficients in $\mathfrak{R}$, and hence $f_{\xi} \in T_{\tau, k, \xi}\left(\Delta_{N}, \Lambda ; \mathfrak{R}\right)$ if $n>1$. Suppose $n=1$. By (3.18) and (3.19), we have

$$
F^{\beta \cdot \delta}(z, 0, w)=v(\alpha)^{-k s} \tau\left(\begin{array}{cc}
1_{n} & 0 \\
0 & \nu(\beta) 1_{s}
\end{array}\right)^{-1} \sum_{\xi} e_{s}\left(-\frac{1}{2} v(\alpha)^{-1} \xi \cdot{ }^{t} p q\right)\left(f_{\xi}\right)_{*}\left(\Omega_{z} v, z\right)^{\beta} e_{s}\left(\frac{1}{2} v(\alpha)^{-1} \xi w\right)
$$

where ${ }^{t} \alpha v=\left(\begin{array}{l}p \\ q\end{array}\right)$. Since $F^{\beta^{\prime} \delta} \in m_{\tau, k}^{\prime}$, this shows that $\left(f_{\xi}\right)_{*}\left(\Omega_{z} v, z\right)^{\beta}$ is finite at $i \infty$ for every $\beta \in \mathfrak{S}_{\mathbf{Q}}$ and every $v \in \mathbf{Q}_{s}^{2}$. Therefore $f_{\xi} \in T_{\tau, k, \xi}$.

TH TOREM 6.2. There is a unique continuous injective homomorphism $y \mapsto y^{\prime}$ of (S) into (S)' which coincides with $\iota_{1}(t) \mapsto \iota_{1}^{\prime}(t)$ on $\iota_{1}\left(\mathbf{A}_{+}^{\times}\right)$and with $(6.11)$ on $\mathbb{S G}_{\mathbf{Q}}$, and which makes the diagram

5-782901 Acta mathematica 141. Imprimé le 1 Septembre 1978 


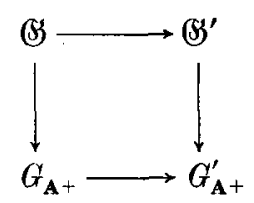

commutative. Moreover, if $F$ is an element of $m_{\tau, k}^{\prime}\left(\mathbf{Q}_{\mathrm{ab}}\right)$ with expansion (6.4), then, for every $y \in(S)$, we have

$$
F^{y^{\prime}}(z, u, w)=|v(y)|^{-k s} \sum_{\xi}\left(f_{\xi}\right)^{y}(u, z) e_{s}\left(\frac{1}{2}|v(y)|^{-1} \xi w\right)
$$

where $\left(f_{\xi}\right)^{y}$ is defined as in Theorem 3.10. If $k \in \mathbf{Z},(6.15)$ holds with $y \in G_{\mathbf{A}+}$ and $y^{\prime} \in G_{\mathbf{A}+}^{\prime}$.

Proof. If $y=(\alpha, \psi)$ and $y^{\prime}=\left(\alpha^{\prime}, \psi^{\prime}\right)$ as in (6.11), we obtain (6.15) from (6.14). Next $(6.15)$ is obvious if $y=\iota_{1}(t)$ and $y^{\prime}=\iota_{1}^{\prime}(t)$ with $t \in \mathbf{Z}_{\mathrm{f}}^{\times}$. Now, given $y=(x, v) \in\left(\mathbb{S}\right.$, an element $y^{\prime}$ of $\left(G^{\prime}\right.$ with projection $x^{\prime}$ on $G_{\mathbf{A}^{+}}^{\prime}$ is uniquely determined by (6.15), if it exists. Let $\mathfrak{P}$ be the subgroup of $\left(\mathfrak{G}\right.$ generated by $\iota_{1}\left(\mathbf{Z}_{\mathfrak{f}}^{\times}\right), G_{\infty+}$, and $\mathfrak{G}_{\mathbf{Q}}$. Then we can define an injective homomorphism $y \mapsto y^{\prime}$ of $\mathfrak{B}$ into $\mathscr{B S}^{\prime}$ which satisfies (6.15) and makes the diagram

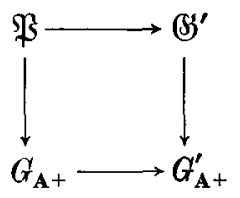

commutative. This homomorphism is continuous, since a basis of neighborhoods of the identity element of $\left(\mathfrak{S}\right.$ can be given by (2.9). By Prop. 2.2, $\mathfrak{P}$ is dense in $\mathfrak{G S}^{3}$, and $\mathfrak{G}^{\prime}$ ' is locally compact, and hence the map can be extended to the whole $\mathfrak{G S}$ as desired.

In the above, we assumed $n>0$, but the ordinary Fourier expansion $F(w)=\sum_{\xi} f_{\xi} e_{s}(\xi w)$ of a modular form $F$ on $\mathfrak{H}_{s}$ with $f_{\xi} \in \mathbf{C}$ may be regarded as the extreme case $n=0$. Then (2.6) or (1.10) may be considered the extreme case of (6.15) with the map $\iota$ of $\mathbf{A}^{\times}$into $G_{\mathbf{A}}^{\prime}$ in place of the injection $G_{\mathbf{A}} \rightarrow G_{\mathbf{A}}^{\prime}$.

\section{Concluding remarks}

A more informative title of this section may be "What Theorems 3.10 and 6.2 suggest in the general theory of arithmetic automorphic forms". Let $G$ be a $\mathbf{Q}$-simple algebraic group such that $G_{\mathbf{R}}$ modulo a maximal compact subgroup is a symmetric domain $\mathbb{S}$, and let $\Gamma$ be a congruence subgroup of $G_{\mathbf{Q}}$. Our main interest here is in the nature of the Fourier expansion of an automorphic form on $\subseteq$ with respect to $\Gamma$, when $\subseteq / \Gamma$ is not compact. If $\subseteq$ is a tube domain and $G$ has a sufficiently large subgroup acting on $\subseteq$ as a group 
of translations, an automorphic form has a Fourier expansion similar to the expansion (1.3) of a Siegel modular form. In general, however, $\subseteq$ may not be a tube domain; even if $\subseteq$ is a tube domain, the group of translations may not be large enough to guarantee such an expansion. In order to study this point in detail, Pyatetskii-Shapiro introduced in [3] the notion of Siegel domains of the first, second, and third kinds, and discussed a certain Fourier expansion for an automorphic form, which he called a Fourier-Jacobi series. When $\subseteq$ is represented as a domain of the third kind, the series has the form

$$
\sum_{\lambda \in L} g_{\lambda}(u, z) e(\langle\lambda, w\rangle)
$$

where $\langle$,$\rangle is a C-bilinear form in a certain complex vector space, g_{\lambda}(u, z)$ is a theta function in $u \in \mathbf{C}^{m}$ with a parameter $z$, and $\lambda$ runs over a lattice $L$. Expansion (6.4) is actually an example of (7.1). If $\subseteq$ is of the first or second kind, $g_{\lambda}(u, z)$ is just a constant, or a function only in $u$ without $z$. Now suppose that we can construct canonical models with respect to $G$, or rather, with respect to a reductive group containing $G$, and therefore can speak of arithmetic automorphic functions with respect to $\Gamma$. Then we may naturally ask the following questions.

(I) Can one define "arithmetic automorphic forms" consistent with the notion of canonical models and arithmetic automorphic functions?

This question can be asked even in the case of compact quotient.

(II) Assuming the quotient non-compact, can one characterize such arithmetic forms in terms of the properties of the Fourier coefficients $g_{\lambda}$ of $(\% .1)$ ?

(III) Are holomorphic Eisenstein series, up to constant factors, arithmetic?

The answers to all these questions seem to be in the affirmative. For example, the results of our present and previous papers give affirmative answers to (I) and (II) for the symplectic groups. The works of Siegel, Klingen, Baily and others answer (III) positively for many groups $\Gamma$ when the Fourier coefficients are constants. In general, if the Fourier coefficients are constants, it is reasonable to call (7.1) arithmetic when the $g_{\lambda}$ belong to $\mathbf{Q}_{\mathrm{ab}}$.

Now, in the case in which $\subseteq$ is of the third kind, the answers to (I), (II) will probably be given in the following way. First, the parameter $z$ belongs to a symmetric domain $\subseteq_{0}$ on which an algebraic group $G_{0}$ acts. There is an injection of $G_{0}$ into a symplectic group which induces a holomorphic embedding $\varepsilon$ of $\mathfrak{S}_{0}$ into $\mathfrak{\mathfrak { S }}_{n}$ for some $n$. Then $g_{\lambda}(u, z)$, possibly modified by a suitable factor, is a function on $\mathbf{C}^{m} \times \mathfrak{S}_{0}$ whose behavior is similar to $h(u, \varepsilon(z))$ with an element $h$ of $T_{k, \xi}$ of $\S 3$. In other words, if $z$ is a "generic point" of $\Im_{0}$ and $A$ is an abelian variety associated with the point $\varepsilon(z)$, then $g_{\lambda}$ as a function in $u$ is a theta function of $A$, behaving like $\theta(u, \varepsilon(z) ; r, s)$. Define $\left(g_{\lambda}\right)_{*}$ in the same manner as in 
$\S 3$. Then we call (7.1) arithmetic if the values $\left(g_{\lambda}\right)_{*}(v, z)$ for all $\lambda$ and all $v$ commensurable with the periods are arithmetic automorphic forms in $z \in \mathfrak{S}_{0}$. Thus question (II) for $G$ can be reduced to question (I) for $G_{0}$, which is comparatively easy. The quotient of $\mathfrak{S}_{0}$ by a discontinuous subgroup of $G_{0}$ may be compact. For example, let $G$ be the unitary group of a hermitian form over a totally indefinite quaternion algebra whose center is totally real. Then a natural choice of a parabolic subgroup yields Fourier expansions in a domain of the first or third kind according as the degree of the hermitian form is even or odd. A recent work of Garrett [2] gives affirmative answers to questions (I) and (II) in either case, endorsing what we said above.

In general, it is expected that $A$ is a generic member of a family of abelian varieties characterized by their endomorphism algebras, or the product of several copies of such. Then the action of the adelization of $G_{0}$ on $g_{\lambda}$ is essentially obtained as a specialization of Theorem 3.10, in a fashion similar to that explained at the end of $\S 3$. Also, one should be able to prove an analogue of Theorem 6.2 with $\subseteq$ and $\mathfrak{S}_{0}$ in place of $\mathfrak{H}_{n+s}$ and $\mathfrak{h}_{n}$.

The domain $\mathfrak{S}$ of the second kind may be viewed as an extreme case where $\mathfrak{S}_{0}$ consists of a single point; the behavior of $g_{\lambda}$ in this case is similar to $h^{*}$ with $h \in T_{k, \xi}$. A typical example of $G$ with such an $\mathfrak{S}$ is provided by the unitary group of a hermitian form over an imaginary quadratic field, which we treated in [9]. The answers to questions (I) and (II) can be given exactly in the above described fashion. The domain of the second kind occurs also when $G$ is the unitary group of a skew-hermitian form over a definite quaternion algebra. As for question (III), we indicated in the same article [9], if without details, that the answer is affirmative at least if $\mathfrak{S}$ is a complex unit ball

$$
\left\{\left.\left(z_{1}, \ldots, z_{n}\right) \in \mathbf{C}_{n}^{1}\left|\sum_{\nu=1}^{n}\right| z_{\nu}\right|^{2}<1\right\} .
$$

It is very likely that the same holds in a more general case.

\section{Appendix}

Our purpose here is to determine the constant factor in the transformation formula of the function $\theta(u, z ; r, s)$ defined by (3.26). To simplify our notation, for every symmetric matrix $S$, let $\{S\}$ denote the column vector consisting of the diagonal elements of $S$; also put $S[X]={ }^{t} X S X$ for a matrix $X$ such that $S X$ can be defined. Let a subgroup $\Gamma_{\theta}$ of $\Gamma_{1}$ be defined by

$$
\Gamma_{\theta}=\left\{\left(\begin{array}{ll}
a & b \\
c & d
\end{array}\right) \in \Gamma_{1} \mid\left\{{ }^{t} a c\right\} \equiv\left\{{ }^{t} b d\right\} \equiv 0\left(\bmod 2 \mathbf{Z}^{n}\right)\right\} \quad \text { (cf. [8, §l]) }
$$


For every $\gamma=\left(\begin{array}{ll}a & b \\ c & d\end{array}\right) \in \Gamma_{\theta}$, we have

$\theta\left({ }^{t}(c z+d)^{-1} u, \gamma(z) ; g, h\right)=\lambda(\gamma) e\left(\left({ }^{t} g h-{ }^{t} g^{*} h^{*}\right) / 2\right) \operatorname{det}(c z+d)^{1 / 2} e\left(\frac{1}{2} \cdot{ }^{t} u(c z+d)^{-1} c u\right) \theta\left(u, z ; g^{*}, h^{*}\right)$,

where $\lambda(\gamma)$ is a constant depending only on $\gamma$, and

$$
\left(\begin{array}{l}
g^{*} \\
h^{*}
\end{array}\right)=t_{\gamma}\left(\begin{array}{l}
g \\
h
\end{array}\right)
$$

This is classical; for a short proof, see [7]. Define $\varphi$ and $\varphi^{\prime}$ by (3.27) and (3.28). Then formula (a.2) is equivalent with

$$
\varphi^{\prime}\left({ }^{t}(c z+d)^{-1} u, \gamma(z) ; g, h\right)=\lambda(\gamma) e\left(\left({ }^{t} g h-{ }^{t} g^{*} h^{*}\right) / 2\right) \operatorname{det}(c z+d)^{1 / 2} \varphi^{\prime}\left(u, z ; g^{*}, h^{*}\right)
$$

This holds also with $\varphi$ instead of $\varphi^{\prime}$.

Proposition A.1. If $\gamma=\left(\begin{array}{ll}a & b \\ c & d\end{array}\right) \in \Gamma_{\theta}, \operatorname{det}(d)>0$, and $\operatorname{det}(c z+d)^{1 / 2}$ is chosen so as to become positive when $z=$ iy with real $y$ tends to 0 , then $\lambda(\gamma)=\operatorname{det}(d)^{-1 / 2} \sum_{v \in V} e\left(b d^{-1}[v] / 2\right)$, where $V$ is a complete set of representatives for $\mathbf{Z}^{n} / d \mathbf{Z}^{n}$.

Proof. Put $\theta(z)=\theta(0, z ; 0,0), w={ }^{t} d^{-1} z(c z+d)^{-1}, f=b d^{-1}$. Then $\gamma(z)=w+f$, so that

$$
\lambda(\gamma) \operatorname{det}(c z+d)^{1 / 2} \theta(z)=\theta(\gamma(z))=\theta(w+f)=\sum_{x \in \mathbf{Z}^{n}} e\left(\frac{1}{2}(f+w)[x]\right)
$$

With $V$ as above, put $x=v+d s$ with $v \in V$ and $s \in \mathbf{Z}^{n}$. Then

$$
\begin{aligned}
\theta(w+f) & =\sum_{v} \sum_{s} e\left(\frac{1}{2} f[v+d s]+\frac{1}{2} w[v+d s]\right) \\
& =\sum_{v} e\left(\frac{1}{2} f[v]\right) \theta\left(0, z(c z+d)^{-1} d ; d^{-1} v, 0\right),
\end{aligned}
$$

since $f[v+d s] / 2 \equiv f[v] / 2(\bmod \mathbf{Z})$. Now, from $\left[7,\left(16^{\prime}\right)\right]$, we obtain

$$
\operatorname{det}(-i z)^{1 / 2} \theta(0, z ; g, h)=e\left({ }^{t} g h\right) \theta\left(0,-z^{-1} ;-h, g\right) .
$$

Put $z=i \tau 1_{n}$ with $0<\tau \in \mathbf{R}$, and observe that

$$
\lim _{\tau \rightarrow 0} \tau^{n / 2} \theta\left(0, i \tau \mathbf{1}_{n} ; g, h\right)=e\left({ }^{t} g h\right) \delta(h),
$$

where $\delta(h)=1$ or 0 according as $h \in \mathbf{Z}^{n}$ or $h \notin \mathbf{Z}^{n}$. Taking the limit of $\tau^{n / 2}$ times (a.4) when $\tau$ tends to 0 , we obtain the desired formula for $\lambda(\gamma)$. 
Proposition A.2. Let $\sigma$ be a symmetric element of $\mathbf{Z}_{n}^{n} \cap G L_{n}(\mathbf{Q})$, and let $\left(\begin{array}{ll}p & q \\ r & s\end{array}\right) \in S L_{2}(\mathbf{Z})$. Suppose that $0<s \equiv 1(\bmod 2), r \sigma^{-1} \in \mathbf{Z}_{n}^{n}, r \neq 0$, and $\left\{p r \sigma^{-1}\right\} \equiv\{q s \sigma\} \equiv 0\left(\bmod 2 \mathbf{Z}^{n}\right)$. Then

$$
\lambda\left(\begin{array}{cc}
p 1_{n} & q \sigma \\
r \sigma^{-1} & s 1_{n}
\end{array}\right)=\varepsilon_{s}^{n}\left(\frac{-2 r}{s}\right)^{n}\left(\frac{\operatorname{det}(\sigma)}{s}\right)
$$

where $\varepsilon_{s}=1$ or $i$ according as $s \equiv 1$ or $-1(\bmod 4)$, and $(-)$ is the quadratic residue symbol.

Proof. Substituting $z+h$ for $z$ in (a.2) with $h={ }^{t} h \in \mathbf{Z}_{n}^{n}$ such that $\{h\} \in 2 \mathbf{Z}^{n}$, we find

$$
\lambda\left(\begin{array}{ll}
a & b+a h \\
c & d+c h
\end{array}\right)=\lambda\left(\begin{array}{ll}
a & b \\
c & d
\end{array}\right)
$$

Since $(2 r, s)=1$, there is a prime $l$, not dividing $\operatorname{det}(\sigma)$, of the form $l=8 m r+s$ with $0<m \in \mathbf{Z}$. Put $k=8 m p+q$. By (a.6) and Prop. A.1, we have

$$
\lambda\left(\begin{array}{cc}
p \mathbf{1}_{n} & q \sigma \\
r \sigma^{-1} & s \mathbf{1}_{n}
\end{array}\right)=\lambda\left(\begin{array}{cc}
p \mathbf{1}_{n} & k \sigma \\
r \sigma^{-1} & l 1_{n}
\end{array}\right)=l^{-n / 2} \sum_{v \in V} e\left(\frac{k}{2 l} \sigma[v]\right)
$$

where $V$ is a complete set of representatives for $\mathbf{Z}^{n} / l \mathbf{Z}^{n}$. Now we can find an element $\alpha$ of $G L_{n}(\mathbf{Q}) \cap \mathbf{Z}_{n}^{n}$ such that $l$ does not divide $\operatorname{det}(\alpha)$ and ${ }^{t} \alpha \sigma \alpha$ is congruent to a diagonal matrix modulo $l$. Let $\xi_{1}, \ldots, \xi_{n}$ be the diagonal elements of ${ }^{t} \alpha \sigma \alpha$. Then (a.7) is equal to

$$
l^{-n / 2} \prod_{v=1}^{n}\left\{\sum_{x=1}^{l} e\left(\frac{k \xi_{v}}{2 l} x^{2}\right)\right\}=\prod_{v=1}^{n} \varepsilon_{l}\left(\frac{2 k \xi_{v}}{l}\right)=\varepsilon_{l}^{n}\left(\frac{2 k}{l}\right)^{n}\left(\frac{\operatorname{det}(\sigma)}{l}\right) .
$$

Since $\left(\begin{array}{ll}p & k \\ r & l\end{array}\right) \in S L_{2}(Z)$, we have $k r \equiv-1(\bmod l)$. Also we have $l \equiv s(\bmod 8 r)$, so that $\varepsilon_{l}=\varepsilon_{s}$, and $\left(\frac{2 k}{l}\right)=\left(\frac{-2 r}{l}\right)=\left(\frac{-2 r}{s}\right)$. Since $r^{n} \operatorname{det}(\sigma)^{-1}$ is an integer, $\operatorname{det}(\sigma)$ is a square times a divisor of $r$, and hence $\left(\frac{\operatorname{det}(\sigma)}{l}\right)=\left(\frac{\operatorname{det}(\sigma)}{s}\right)$, which completes the proof.

\section{References}

[1]. Cartan, H., Formes modulaires. Séminaire H. Cartan 1957/58, exp. 4.

[2]. GarRetr, P., Arithmetic automorphic forms for quaternion unitary groups. Thesis, Princeton University, 1977.

[3]. Pyatetskm-ShapIro, I. I., Geometry of classical domains and the theory of automorphic functions. Moscow, 1961 (Russian).

[4]. Shrmura, G., On canonical models of arithmetic quotients of bounded symmetric domains, I, Il. Ann. of Math., 91 (1970), 144-222; 92 (1970), 528-549. 
[5]. - On some arithmetic properties of modular forms of one and several variables. Ann. of Math., 102 (1975), 491-515.

[6]. - On the Fourier coefficients of modular forms of several variables. Göttingen Nachr. Akad. Wiss., 1975, 261-268.

[7]. — Theta functions with complex multiplication. Duke Math. J., 43 (1976), 673-696.

[8]. - On the derivatives of theta functions and modular forms. Duke Math. J., 44 (1977), 365-387.

[9]. - Unitary groups and theta functions. Proceedings of Int. Symp. on Alg. N. Th. Kyoto 1976 (1977), 195-200.

[10]. WeIL, A., Sur certains groupes d'opérateurs unitaires. Acta Math., 111 (1964), 143-211.

Received June 20, 1977 